\title{
Three-dimensional interferometric, spectrometric, and planetary views of Procyon
}

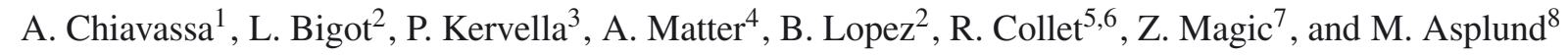 \\ 1 Institut d'Astronomie et d'Astrophysique, Université Libre de Bruxelles, CP. 226, Boulevard du Triomphe, 1050 Bruxelles, Belgium \\ e-mail: achiavas@ulb.ac.be \\ ${ }^{2}$ Université de Nice Sophia-Antipolis, Observatoire de la Côte d'Azur, CNRS Laboratoire Lagrange, BP 4229, 06304 Nice Cedex 4, \\ France \\ 3 LESIA, Observatoire de Paris, CNRS UMR 8109, UPMC, Université Paris Diderot, 5 place Jules Janssen, 92195 Meudon, France \\ ${ }^{4}$ Max-Planck-Institut für Radioastronomie, Auf dem Hügel 69, 53121 Bonn, Germany \\ 5 Centre for Star and Planet Formation, Natural History Museum of Denmark University of Copenhagen, Øster Voldgade 5-7, \\ 1350 Copenhagen, Denmark \\ 6 Astronomical Observatory/Niels Bohr Institute, Juliane Maries Vej 30, 2100 Copenhagen, Denmark \\ 7 Max Planck Institute for Astrophysics, Karl-Schwarzschild-Str. 1, 85741 Garching, Germany \\ ${ }^{8}$ Research School of Astronomy and Astrophysics, Australian National University, Cotter Rd., Weston Creek, ACT 2611, Australia
}

Received 15 December 2011 / Accepted 16 January 2012

\begin{abstract}
Context. Procyon is one of the brightest stars in the sky and one of our nearest neighbours. It is therefore an ideal benchmark object for stellar astrophysics studies using interferometric, spectroscopic, and asteroseismic techniques.

Aims. We use a new realistic three-dimensional (3D) radiative-hydrodynamical (RHD) model atmosphere of Procyon generated with the STAGGer Code and synthetic spectra computed with the radiative transfer code Optim3D to re-analyze interferometric and spectroscopic data from the optical to the infrared. We provide synthetic interferometric observables that can be validated using observations. Methods. We computed intensity maps from a RHD simulation in two optical filters centered on 500 and $800 \mathrm{~nm}$ (MARK III) and one infrared filter centered on $2.2 \mu \mathrm{m}$ (VINCI). We constructed stellar disk images accounting for the center-to-limb variations and used them to derive visibility amplitudes and closure phases. We also computed the spatially and temporally averaged synthetic spectrum from the ultraviolet to the infrared. We compare these observables to Procyon data.

Results. We study the impact of the granulation pattern on center-to-limb intensity profiles and provide limb-darkening coefficients in the optical as well as in the infrared. We show how the convection-related surface structures affect the visibility curves and closure phases with clear deviations from circular symmetry, from the 3rd lobe on. These deviations are detectable with current interferometers using closure phases. We derive new angular diameters at different wavelengths with two independent methods based on 3D simulations. We find that $\theta_{\text {Vinci }}=5.390 \pm 0.03$ mas, which we confirm by comparison with an independent asteroseismic estimation $\left(\theta_{\text {seismic }}=5.360 \pm 0.07\right.$ mas. The resulting $T_{\text {eff }}$ is $6591 \mathrm{~K}$ (or $6556 \mathrm{~K}$ depending on the bolometric flux used), which is consistent with the value of $T_{\text {eff,IR }}=6621 \mathrm{~K}$ found with the infrared flux method. We measure a surface gravity $\log g=4.01 \pm 0.03\left[\mathrm{~cm} / \mathrm{s}^{2}\right]$ that is higher by 0.05 dex than literature values. Spectrophotometric comparisons with observations provide very good agreement with the spectral energy distribution and photometric colors, allowing us to conclude that the thermal gradient in the simulation matches Procyon fairly well. Finally, we show that the granulation pattern of a planet-hosting Procyon-like star has a non-negligible impact on the detection of hot Jupiters in the infrared using interferometry closure phases. It is then crucial to have a comprehensive knowledge of the host star to directly detect and characterize hot Jupiters. In this respect, RHD simulations are very important to achieving this aim.
\end{abstract}

Key words. radiative transfer - hydrodynamics - techniques: interferometric - planetary systems - stars: atmospheres stars: individual: Procyon

\section{Introduction}

Procyon ( $\alpha$ Canis Minoris) is one of the brightest stars in the sky and one of our nearest neighbours. It is therefore an ideal target for stellar astrophysics studies.

For this reason, it has a long history of observations. Bessel (1844) discovered that its motion was perturbed by an invisible companion. Procyon became, after Sirius, one of the first astrometric binaries ever detected. The first orbital elements were determined by Auwers (1862), who showed that the period of revolution is about 40 years. The faint companion, Procyon B, was not detected visually until the end of the nineteenth century by Schaeberle (1896). It was one of the first detected white dwarfs
(Eggen \& Greenstein 1965). The main component of the system is a subgiant F5 IV-V (Procyon A, HR 2943, HD 61421) that is ending its life on the main sequence (Eggenberger et al. 2005; Provost et al. 2006). It has a solar metallicity (Griffin 1971; Steffen 1985; Allende Prieto et al. 2002) with an effective temperature around $T_{\text {eff }} \approx 6500 \mathrm{~K}$ (Code et al. 1976). One of the first radius determinations was made photometrically by Gray (1967), who found that $R=2.24 R_{\odot}$. The binary nature of the system is a great opportunity to determine the mass of both companions. The first attempt was made by Strand (1951), who determined the masses of Procyon A and B by determining the parallax and orbital elements of the system. He found that $1.76 \pm 0.1$ and $0.65 \pm 0.05 M_{\odot}$, respectively. 
Steffen (1985) claimed that the mass of Procyon A was too high to be compatible with its luminosity and suggested instead a mass around $1.4 M_{\odot}$. More recent and more accurate determinations of the mass of Procyon A converge to that of a star of either $M=1.497 \pm 0.037 M_{\odot}$ (Girard et al. 2000) or $1.430 \pm 0.0034 M_{\odot}$ (Gatewood \& Han 2006). The age of the system is well-constrained by the white dwarf companion, whose cooling law as a function of time is well-established. Provencal et al. (2002) found an old white dwarf with an age of 1.7 $\pm 0.1 \mathrm{~Gy}$. This determination is a strong constraint on stellar evolution models.

A way to discriminate between different masses and ages is to determine the interferometric radius. The first attempt to measure the diameter of Procyon was made by Hanbury Brown et al. (1967, 1974), who found an angular diameter of $\theta=$ $5.50 \pm 0.17$ mas. This value was confirmed later by Mozurkewich et al. (1991) but with a much higher precision $(\sim 1 \%)$. More recently, Kervella et al. (2004b) redetermined the angular diameter using the VINCr instrument at VLTI. They found a diameter that is even smaller $\theta=5.448 \pm 0.053$ mas. Aufdenberg et al. (2005) reanalyzed these data using hydrodynamical model atmospheres and found that $\theta=5.404 \pm 0.031$ mas (see their Table 7). It is interesting to compare these angular diameters with those derived using the independent infrared flux method. A recent determination was performed by Casagrande et al. (2010), who derived a value of $\theta_{\mathrm{IR}}=5.326 \pm 0.068$ mas that is smaller than the Kervella et al.'s result but in agreement to within $1 \sigma$.

Another important characteristic of Procyon is the presence of oscillations caused by trapped acoustic modes. The first claims of a detection of an excess power were made by Gelly et al. (1986, 1988) and Brown et al. (1991), who found a mean large spacing between consecutive acoustic modes of about $39 \mu \mathrm{Hz}$ and $55 \mu \mathrm{Hz}$, respectively. Martić et al. (1999) made a clear detection using the ELODIE fiber échelle spectrograph with a strong excess power around $\nu_{\max } \approx 1000 \mu \mathrm{Hz}$ and confirmed the results of Brown et al. (1991). They determined the frequency spacing to be $55 \mu \mathrm{Hz}$. Later, Eggenberger et al. (2004) and Martić et al. (2004) made the first identifications of individual frequencies of spherical harmonic degrees $\ell=0,1,2$ with mean large spacings of $55.5 \pm 0.5$ (Eggenberger et al. 2004) and $53.5 \pm 0.5 \mu \mathrm{Hz}$ (Martić et al. 2004). More recent observations were made from the ground during single or multisite campaigns (Mosser et al. 2008; Arentoft et al. 2008; Bedding et al. 2010) or from space onboard the MOST satellite (Matthews et al. 2004; Guenther et al. 2008), which considerably improved the precision in frequency to within $\sim 1 \mu \mathrm{Hz}$.

A stellar evolution model of Procyon was developed by Hartmann et al. (1975), who used the astrometric mass, photometry, and the angular diameter of Hanbury Brown et al. (1974) to constrain the model. More realistic modeling, especially with more precise equation-of-states, came with Guenther $\&$ Demarque (1993), who demonstrated the importance of diffusion of elements. The values of individual p-modes frequencies tightly constrained the measurements of the fundamental parameters (Barban et al. 1999; di Mauro \& Christensen-Dalsgaard 2001; Eggenberger et al. 2005; Provost et al. 2006; Bonanno et al. 2007; Guenther et al. 2008). Eggenberger et al. (2005) and Provost et al. (2006) presented a realistic stellar evolution modeling to constrain simultaneously the location in the HerzsprungRussell (HR) diagram and the large frequency separations of Eggenberger et al. (2004) and Martić et al. (2004), respectively. They disagreed on the derived mass. Eggenberger et al. found a mass that agrees well with the astrometric value derived by Girard et al. (2000), whereas Provost et al. found a mass that cor- responds to the more recent value of Gatewood \& Han (2006). Part of the reason for the difference in the two stellar evolution models comes from the slightly different large separation (which increases with the mass), and is partly due to the difference in the stellar evolution codes themselves. We emphasize that the most accurate stellar model of Provost et al. that fits asteroseismic and spectrophotometric data has an age ( $\geq 2 \mathrm{~Gy})$, which is consistent with the age of the white dwarf. The astrometric mass of Procyon B, indeed, implies that the mass of its progenitor was about $3 M_{\odot}$, and that it has lived about $\sim 500$ My on the main sequence. Therefore, we can safely conclude that the age of the system must be at least $2 \mathrm{~Gy}$. The higher mass of Eggenberger et al. (2005) corresponds to a younger ( $\leq 1.7 \mathrm{~Gy})$ system, which is inconsistent with the result of Provencal et al. (2002).

The atmospheric parameters $\left(T_{\text {eff }}, \log g,[\mathrm{Fe} / \mathrm{H}]\right)$ and the interferometric radius, which are used to define the stellar evolution model and the analysis of frequencies, depend strongly on the realism of the atmosphere and the exactness of the temperature gradient in the surface layers. In the case of F-stars, these gradients are strongly modified by the convective transport which is more vigorous than in the Sun. This is clearly seen in the line bisectors (Gray 1981; Dravins 1987), which are two or three times larger than in the Sun. The larger convective velocities are due to the higher stellar luminosity and lower densities. This strong effect of convection must be taken into account for stellar physics diagnostics. Realistic three-dimensional (3D) time-dependent hydrodynamical simulations of the surface layer of Procyon were performed by several authors (Atroshchenko et al. 1989; Nordlund \& Dravins 1990; Allende Prieto et al. 2002), who showed that the $3 \mathrm{D}$ effects on such a F-star can significantly affect the line profile formation and abundance analysis (Allende Prieto et al. 2002). Nelson (1980) and Nordlund \& Dravins (1990) showed that the surface defined at $T=T_{\text {eff }}$ is not flat but rather "corrugated" owing to the large fluctuations and the high contrast of granulation. These hydrodynamical simulations can reproduce with success the line shifts, asymmetries, and in particular the observed bisectors of various lines (Allende Prieto et al. 2002, FeI and FeII). Allende Prieto et al. also showed that the $3 \mathrm{D}$ limb darkening law differs significantly from the one-dimensional (1D) law, by up to $\sim 1.6 \%$, leading to a correction of $\Delta T_{\mathrm{eff}} \approx 50 \mathrm{~K}$, which is non-negligible for precise stellar evolution modeling. Aufdenberg et al. (2005) developed 3D models using the CO ${ }^{5}$ BOLD code (Freytag et al. 2002, 2012) to calculate limb-darkened intensity profiles to analyze the visibility curves obtained by the VINCI instrument (Kervella et al. 2003b) and MARK III. Their 3D analysis inferred a radius smaller by 0.04 mas than that of Kervella et al. (2003b), who used a 1D limb-darkened law. Aufdenberg et al. also showed that 3D models accurately reproduce the spectral energy distribution (SED) in the UV, whereas a 1D model was unable to.

To establish the importance of a realistic hydrodynamical modeling of the atmosphere of Procyon, we re-analyze the interferometric and spectroscopic data at the different wavelengths of Aufdenberg et al. (2005) using up-to-date line and continuum opacities (Gustafsson et al. 2008) to derive a new radius. We propose a solution that agrees well with results derived using the astrometric, asteroseismologic, infrared flux method, and interferometric data. We also explore the impact of convectionrelated surface structures on the closure phases and assess how the direct search for planets using interferometry may be affected by the host-star surface structures. 
Table 1. 3D simulation of Procyon used in this work.

\begin{tabular}{lccccc}
\hline \hline $\begin{array}{l}\left\langle T_{\text {eff }}\right\rangle^{a} \\
{[\mathrm{~K}]}\end{array}$ & {$[\mathrm{Fe} / \mathrm{H}]$} & $\begin{array}{c}\log g \\
{\left[\mathrm{~cm} / \mathrm{s}^{2}\right]}\end{array}$ & $\begin{array}{c}x, y, z \text {-dimensions } \\
{[\mathrm{Mm}]}\end{array}$ & $\begin{array}{c}x, y, z \text {-resolution } \\
\text { [grid points] }\end{array}$ & $\begin{array}{c}R_{\star} \\
{\left[R_{\odot}\right]}\end{array}$ \\
\hline $6512 \pm 25^{b}$ & $0.0^{c}$ & 4.0 & $22.0 \times 22.0 \times 17.0$ & $240 \times 240 \times 240$ & $2.055^{d}$ \\
\hline
\end{tabular}

Notes. ${ }^{(a)}$ Horizontally and temporal average and standard deviation of the emergent effective temperatures. ${ }^{(b)}$ Collet et al. (2011). ${ }^{(c)}$ Chemical composition by Asplund et al. (2009). ${ }^{(d)}$ Angular diameter of 5.443 mas (Kervella et al. 2004b) converted into linear radius with Eq. (7).

\section{Three-dimensional radiative-hydrodynamical approach}

\subsection{Procyon simulation}

The convective surface of Procyon is modeled using the STAGGER CoDE (Nordlund et al. 2009; Nordlund \& Galsgaard ${ }^{1}$ ). In a local box located around the optical surface $\tau \approx 1$, the code solves the full set of hydrodynamical equations for the conservation of mass, momentum, and energy coupled to an accurate treatment of the radiative transfer. The equations are solved on a staggered mesh where the thermodynamical scalar variables (density, internal energy, and temperature) are cell centered, while the fluxes are defined on the cell faces. This scheme has several numerical advantages when simulating surface convection. It is robust against shocks and ensures conservation of the thermodynamic variables. The domain of the simulation contains an entropy minimum at the surface that is sufficiently deep for there to be a flat entropy profile at the bottom. The code uses periodic boundary conditions horizontally and open boundaries vertically. At the bottom of the simulation, the inflows have a constant entropy and pressure. The outflows are not tightly constrained and are free to pass through the boundary. The code is based on a sixthorder explicit finite-difference scheme and a, fifth-order interpolation. The numerical viscosity of the Rytchmeyer \& Morton type is used to stabilize the code. The corresponding adjustable parameters are chosen to minimize the viscosity and not adjusted to fit the observables. We used a realistic equation-ofstate that accounts for ionization, recombination, and dissociation (Mihalas et al. 1988) and both continuous (Trampedach et al., priv. comm.) and line opacities (Gustafsson et al. 2008). An accurate treatment of the transfer is needed to get a correct temperature gradient in the transition region between the optically thin and thick layers. The transfer equation is solved using a Feautrier-like scheme along several inclined rays (one vertical, eight inclined) through each grid point. The wavelength dependence of the radiative transfer is taken into account using opacity bins as in Nordlund (1982). The numerical resolution is $240^{3}$. The geometrical sizes are $22 \mathrm{Mm} \times 22 \mathrm{Mm}$ horizontally and $17 \mathrm{Mm}$ vertically. The horizontal dimensions of the box are defined to include at each time step a sufficient number of granules, and the vertical one is chosen to ensure that the entropy profile is flat at the bottom. The equations of magnetohydrodynamics are not computed for this model. The stellar parameters corresponding to our RHD model (Table 1 ) are $T_{\text {eff }}=6512 \pm 25 \mathrm{~K}$, $\log g=4.0\left[\mathrm{~cm} / \mathrm{s}^{2}\right]$, and a solar chemical composition (Asplund et al. 2009). The uncertainty in $T_{\text {eff }}$ represents the fluctuations with time around the mean value. These parameters roughly correspond to those of Procyon. The exact values of the parameters do not influence the limb darkening (Aufdenberg et al. 2005; Bigot et al. 2011).

\footnotetext{
1 1995, http://www. astro.ku.dk/ kg/Papers/MHDcode.ps.gz
}

\subsection{Spherical tiling models, intensity maps, and spectra}

The computational domain of each simulation represents only a small portion of the stellar surface. To obtain an image of the whole stellar disk, we employ the same tiling method explained in Chiavassa et al. (2010a). For this purpose, we used the $3 \mathrm{D}$ pure local thermal equilibrium (LTE) radiative transfer code Optim3D (Chiavassa et al. 2009) to compute intensity maps from the snapshots of the RHD simulation of Table 1 for different inclinations with respect to the vertical, $\mu \equiv \cos (\theta)=[1.000$, $0.989,0.978,0.946,0.913,0.861,0.809,0.739,0.669,0.584$, $0.500,0.404,0.309,0.206,0.104]$ (Fig. 1) and for a representative series of simulation's snapshots: we chose $\sim 25$ snapshots taken at regular intervals and covering $\sim 1 \mathrm{~h}$ of stellar time, which corresponds to $\sim 5 \mathrm{p}$-modes. The code Ортім3D takes into account the Doppler shifts caused by convective motions and the radiative transfer equation is solved monochromatically using pre-tabulated extinction coefficients as functions of temperature, density, and wavelength (with a resolving power of $\lambda / \delta \lambda=500000)$. The lookup tables were computed for the same chemical composition as the RHD simulation (i.e. Asplund et al. 2009) and using the same extensive atomic and molecular opacity data as the latest generation of MARCs models (Gustafsson et al. 2008).

We then used the synthetic images to map onto spherical surfaces accounting for distortions especially at high latitudes and longitudes cropping the square-shaped intensity maps when defining the spherical tiles. Moreover, we selected intensity maps computed from random snapshots in the simulation timeseries: this process avoided the assumption of periodic boundary conditions resulting in a tiled spherical surface displaying globally an artifactual periodic granulation pattern.

On the basis of the stellar radius estimates and the sizes of the simulation domains (Table 1), we required 215 tiles to cover half a circumference from side to side on the sphere (number of tiles $=\pi R_{\star} / 22.0$, where 22.0 is the horizontal dimension of the numerical box in $\mathrm{Mm}$ and $R_{\star}$ the radius of the star). To produce the final stellar disk images, we performed an orthographic projection of the tiled spheres on a plane perpendicular to the lineof-sight $(\mu=1.0)$. The orthographic projection returned images of the globe in which distortions are greatest toward the rim of the hemisphere where distances are compressed (Chiavassa et al. 2010a).

In this work, we computed a synthetic stellar disk image for interferometric spectral bands used in Aufdenberg et al. (2005): (i) the MARK III (Shao et al. 1988) centered at 500 and $800 \mathrm{~nm}$, and (ii) VINCI (Kervella et al. 2003a) centered at $2.2 \mu \mathrm{m}$ (Fig. 2). The MARK III sensitivity curves are assumed to be Gaussian with central wavelengths $\lambda_{0}=500$ and $800 \mathrm{~nm}$, each of them with a FWHM of $20 \mathrm{~nm}$ (Mozurkewich et al. 1991). We produced a number of synthetic stellar disk images corresponding to different wavelengths in the filters with a spectral resolving power of 20000 . Figure 3 shows the resulting synthetic stellar disk images averaged over each passband. 


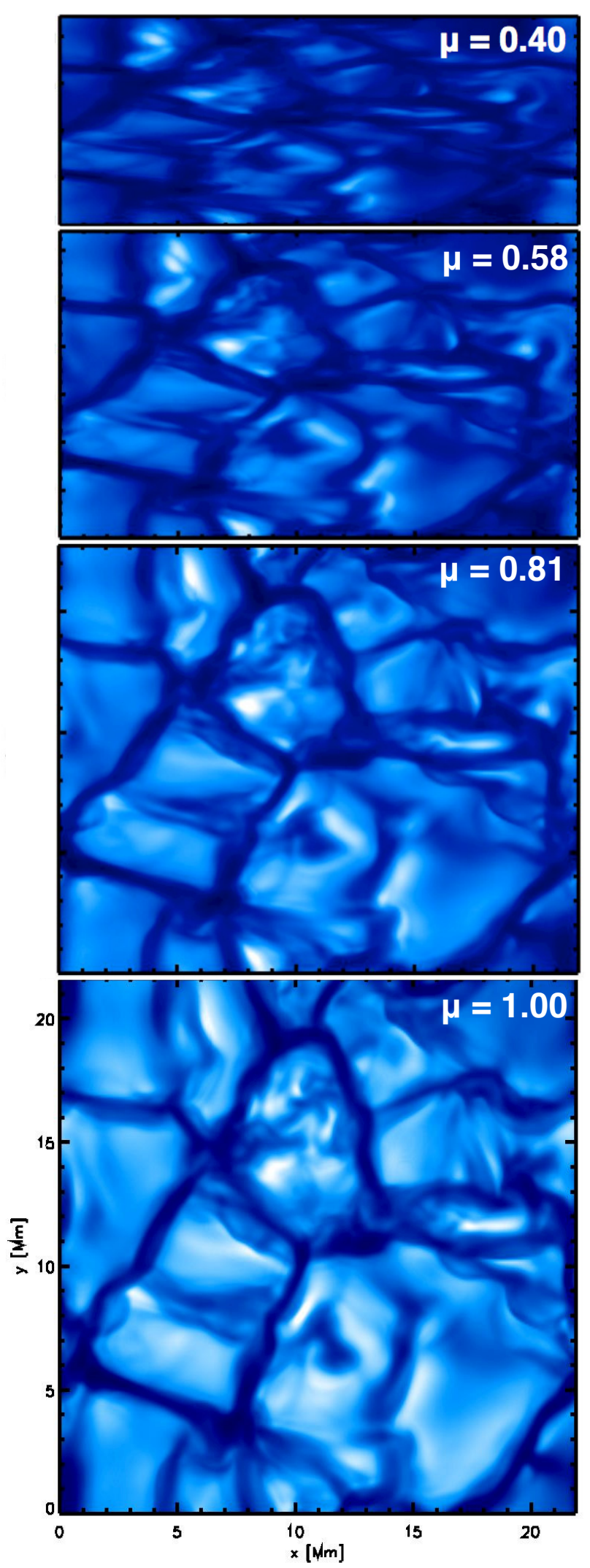

Fig. 1. Intensity maps in the MARK $500 \mathrm{~nm}$ filter for the RHD simulation for different inclination angles $\mu$. The intensity ranges from $1 \times 10^{3}$ to $1.1 \times 10^{7} \mathrm{erg} \mathrm{cm}^{-2} \mathrm{~s}^{-1} \AA^{-1}$.

With Opтiм3D, we also computed the spectra, which were normalized to the filter transmission as $\frac{\int I_{\lambda} T(\lambda) \mathrm{d} \lambda}{\int T(\lambda) \mathrm{d} \lambda}$, where $I_{\lambda}$ is the
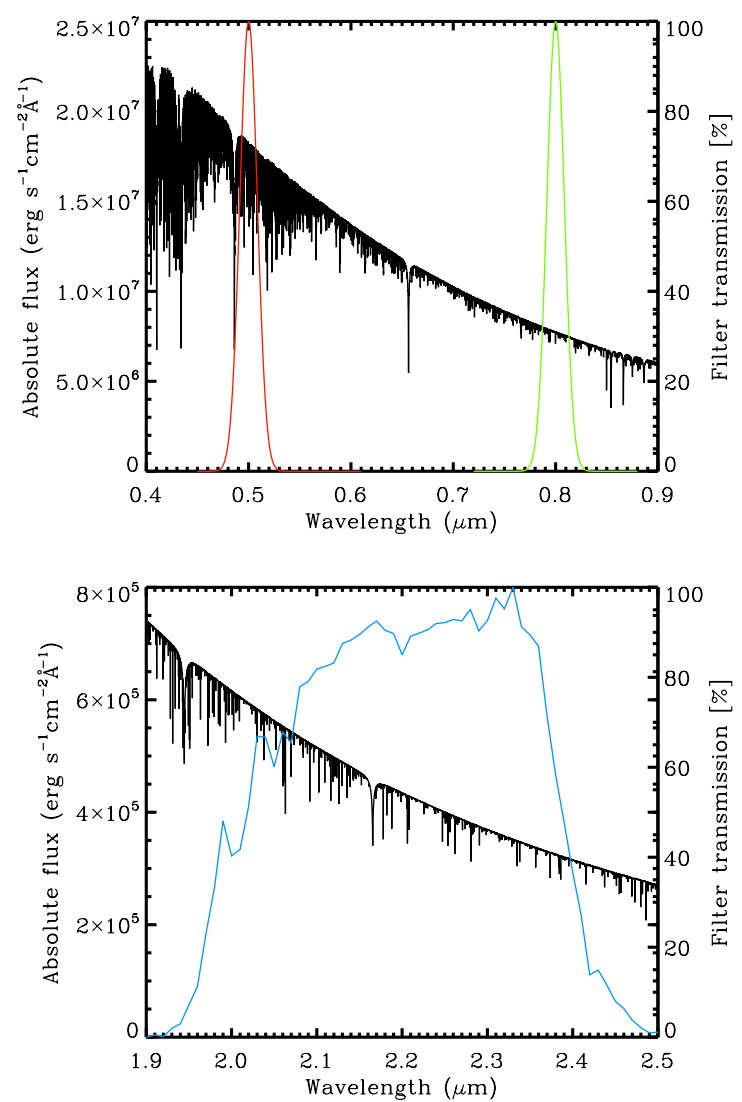

Fig. 2. A spatially and temporally averaged synthetic spectrum in the optical wavelength region with the MARK $500 \mathrm{~nm}$ filter (red: top left), MARK 800 nm (green: top right), and VINCI infrared filter (blue: bottom).

intensity and $T(\lambda)$ is the transmission curve of the filter at a certain wavelength. The spectra in Fig. 2 were computed along rays of four $\mu$-angles $[0.88,0.65,0.55,0.34]$ and four $\phi$-angles $\left[0^{\circ}\right.$, $\left.90^{\circ}, 180^{\circ}, 270^{\circ}\right]$, after which we performed a disk integration and a temporal average over all selected snapshots.

\subsection{Three-dimensional limb-darkening}

Figure 3 shows irregular stellar surfaces with numerous convection-related surface structures. There are pronounced center-to-limb variations in the MARK $500 \mathrm{~nm}$ and MARK $800 \mathrm{~nm}$ filters, which are less noticeable in the VINCI filter, owing manly to the different Planck functions in the optical and the infrared wavelength ranges.

We derived azimuthally averaged (i.e., averaged over different $\phi$ angles) intensity profiles for every synthetic stellar-disk image from the simulation (Fig. 4). Using the method described in Chiavassa et al. $(2009,2010 a)$, the profiles were constructed using rings regularly spaced in $\mu=\cos (\theta)$ for $\mu \leq 1$ (i.e. $\left.r / R_{\star} \leq 1\right)$, with $\theta$ the angle between the line of sight and the vertical direction. The standard deviation in the average intensity, $\sigma_{I(\mu)}$, was computed within each ring, the $\mu$ parameter being connected to the impact parameter $r / R_{\star}$ through the relationship $r / R_{\star}=\sqrt{1-\mu^{2}}$, where $R_{\star}$ is the stellar radius reported in Table 1 . The total number of rings is 20 , which we ensured was sufficiently large for a reliable characterization of the intensity profiles. 
A. Chiavassa et al.: Three-dimensional interferometric, spectrometric, and planetary views of Procyon
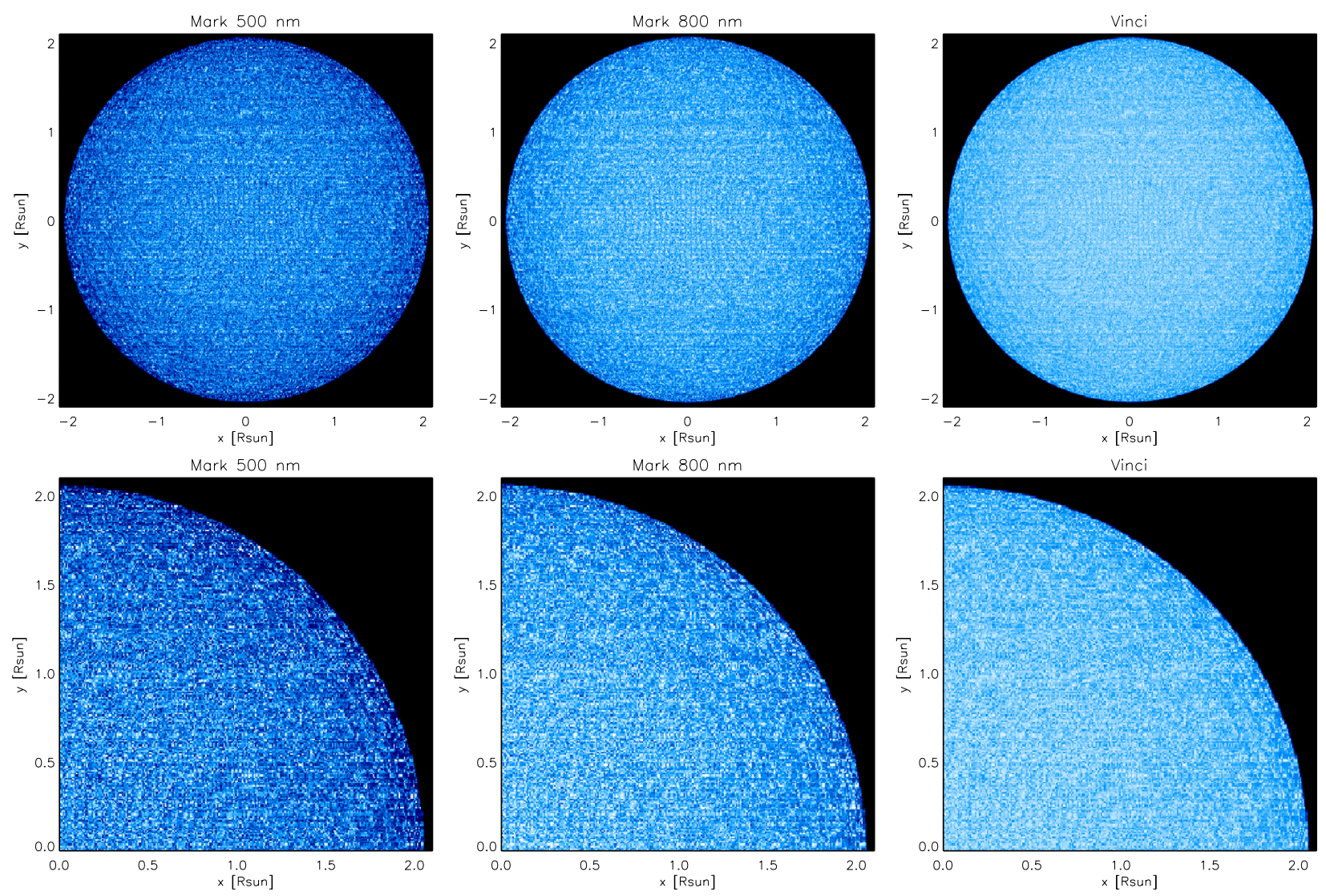

Fig. 3. Top row: synthetic stellar disk images of the RHD simulation. The intensity range is $\left[0.3-1.0 \times 10^{6}\right],\left[0.3-5.2 \times 10^{5}\right]$, and $[0.3-0.9 \times$ $\left.10^{5}\right] \mathrm{erg} \mathrm{cm}^{-2} \mathrm{~s}^{-1} \AA^{-1}$ for MARK $500 \mathrm{~nm}$, MARK $800 \mathrm{~nm}$, and Vinci filters, respectively. Bottom row: enlargements of the images above.
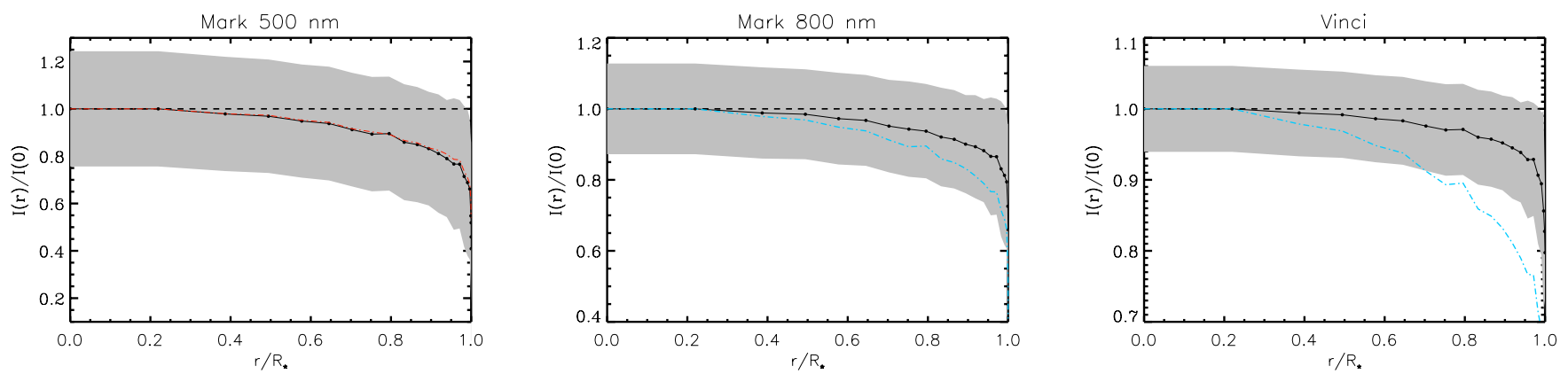

Fig. 4. Radially averaged intensity profiles (black line) derived from the synthetic stellar disk images of Fig. 3. The gray areas denote the $1 \sigma$ spatial fluctuations with respect to the averaged intensity profile. The dashed horizontal line is a uniform disk-intensity profile. The intensity is normalized to the mean intensity at disk center and the radius is normalized to the radius given in Table 1 . The blue dotted-dashed line in the central and right panels is the average intensity profile of MARK $500 \mathrm{~nm}$. The red dotted-dashed line in the left panel is the continuum-only average intensity profile in MARK $500 \mathrm{~nm}$.

Figure 4 displays a steeper center-to-limb variation for the optical region, as already visible in the disk images, with fluctuations of $\sim 20 \%$ in the MARK III $500 \mathrm{~nm}$ filter down to $\sim 10 \%$ and $\sim 5 \%$ in the MARK III $800 \mathrm{~nm}$ and VINCI filters, respectively. We tested the impact of the spectral lines in the MARK $500 \mathrm{~nm}$ filter, for which the effects of lines are stronger, computing a synthetic disk image based only on the continuum opacities. Its average intensity profiles (red line in the left panel of Fig. 4) is very similar to the one computed for spectral lines (black line) with differences smaller than $1 \%$ for $r / R_{\star} \leq 0.9$ and $1-5 \%$ at the limb $\left(0.9<r / R_{\star} \leq 1.0\right)$. We also note that the continuum profile tends to be closer to the uniform disk profile (dashed line in the figure), as well as the MARK $800 \mathrm{~nm}$ and VINCI filters with respect to the intensity profile of the MARK $500 \mathrm{~nm}$.

To fit the average profile, we used the limb-darkening law (Chiavassa et al. 2009):

$$
\frac{I_{\lambda}(\mu)}{I_{\lambda}(1)}=\sum_{k=0}^{N} a_{k}(1-\mu)^{k} .
$$

In this equation, $I_{\lambda}(\mu)$ is the intensity, $a_{k}$ are the limb-darkening coefficients, and $N+1$ their number. We performed a LevenbergMarquardt least squares minimization to fit all the radially averaged profiles of Fig. 4 using this law and weighting the fit 

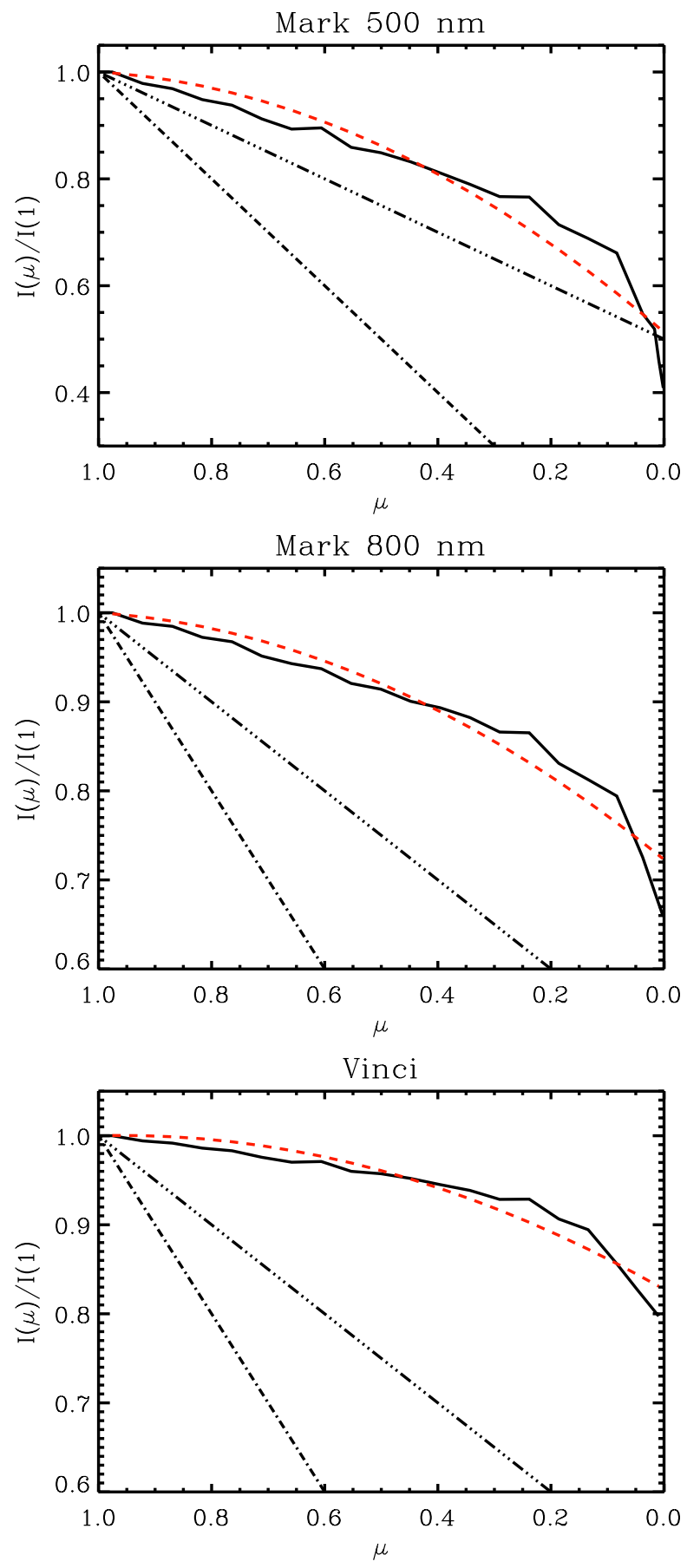

Fig. 5. Center-to-limb fits (red dashed line) obtained using Eq. (1) with $N=2$ for the 3D azimuthally average intensity profile (solid line). A full limb-darkening (dash-dotted line), a partial limb-darkening (triple dot-dashed line) are also shown. $\mu=\cos \theta$, with $\theta$ the angle between the line of sight and the vertical direction.

by $1 / \sigma_{I(\mu)}$, owing to $3 \mathrm{D}$ fluctuations (gray areas of Fig. 4). We varied the order $N$ and found that $N=2$ provides the optimal solution with very minor improvements to the $\chi^{2}$ minimization using $N=3$. This was already found by Chiavassa et al. (2010a) for K giants. Figure 5 shows the fits in the different filters used in this study, while Table 2 reports the limb-darkening coefficients from the fits. The figure illustrates that the average intensity profiles from a RHD simulation differ considerably from the full limb-darkening $(I(\mu) / I(1)=\mu)$ and partial limb-darkening $(I(\mu) / I(1)=0.5 \mu)$ profiles, as well as the power-law profile that
Table 2. Limb-darkening coefficients from the law described in text for RHD simulation of Table 1.

\begin{tabular}{lccc}
\hline \hline Filter & $a_{0}$ & $a_{1}$ & $a_{2}$ \\
\hline MARK 500 nm & 1.000 & -0.066 & -0.421 \\
MARK $800 \mathrm{~nm}$ & 1.000 & -0.041 & -0.236 \\
VINCI & 1.000 & 0.016 & -0.188 \\
\hline
\end{tabular}

does not even provide an appreciable fit to the radially average profile. We therefore discourage the use of these simple laws.

\subsection{Visibility curves}

The synthetic disk images are used to derive interferometric observables. For this purpose, we used the method described in Chiavassa et al. (2009) to calculate the discrete complex Fourier transform $\mathcal{F}$ for each image. The visibility, vis, is defined as the modulus $|\mathcal{F}|$, of the Fourier transform normalized by the value of the modulus at the origin of the frequency plane, $\left|\mathcal{F}_{0}\right|$, with the phase $\tan \varphi=\mathfrak{J}(\mathcal{F}) / \mathfrak{R}(\mathcal{F})$, where $\mathfrak{J}(\mathcal{F})$ and $\mathfrak{R}(\mathcal{F})$ are the imaginary and real parts of the complex number $\mathcal{F}$, respectively. In relatively broad filters, such as VINCI, several spatial frequencies are simultaneously observed by the interferometer. This effect is called bandwidth smearing. Kervella et al. (2003b,c) show that this effect is negligible for squared visibilities larger than $40 \%$ but significant for spatial frequencies close to the first minimum of the visibility function. To account for this effect, we computed the squared visibilities proposed by Wittkowski et al. (2004)

$\left\langle v i s^{2}\right\rangle=\frac{\int_{\lambda_{0}}^{\lambda_{1}} v i s_{\lambda}^{2} \mathrm{~d} \lambda}{\int_{\lambda_{0}}^{\lambda_{1}} T_{\lambda}^{2} F_{\lambda}^{2} \mathrm{~d} \lambda}$,

where $v i s_{\lambda}^{2}$ is the squared visibility at wavelength $\lambda$, both $\lambda_{0}$ and $\lambda_{1}$ are the filter wavelength limits, $T_{\lambda}$ is the transmission curve of the filter, and $F_{\lambda}$ is the flux at wavelength $\lambda$ (see Fig. 2). The value $v i s_{\lambda}^{2}$ was computed from the disk images that already include $T_{\lambda}$ in the calculations.

In our case, we follow these steps:

1. We generate a synthetic stellar disk image at the different wavelengths of the filters;

2. We compute the visibility curves $v i s_{\lambda}^{2}$ for 36 different cuts through the centers of the stellar disk images;

3. We apply Eq. (2) to obtain the average squared visibilities.

Figure 6 (top panels) shows the visibility curves computed with Eq. (2) for 36 different cuts through the centers of synthetic disk images. This is equivalent to generating different realizations of the stellar disk images with intensity maps computed for different sets of randomly selected snapshots. We used a theoretical spatial frequency scale expressed in units of inverse solar radii $\left(R_{\odot}^{-1}\right)$. The conversion between visibilities expressed on the latter scale and the more usual "arcsecond" scale is given by

vis $\left[{ }^{\prime \prime}\right]=\operatorname{vis}\left[R_{\odot}^{-1}\right] \cdot d[\mathrm{pc}] \cdot 214.9$,

where 214.9 is the astronomical unit expressed in solar radius, and $d$ is the distance of the observed star. The spatial frequency in $\operatorname{arcsec}^{-1}$ (i.e., $v$ ) is related to the baseline (i.e., $B$ ) in meters by

$v=\frac{B \cdot 4.84813}{\lambda}$

where $\lambda$ is the wavelength in $\mu \mathrm{m}$. 

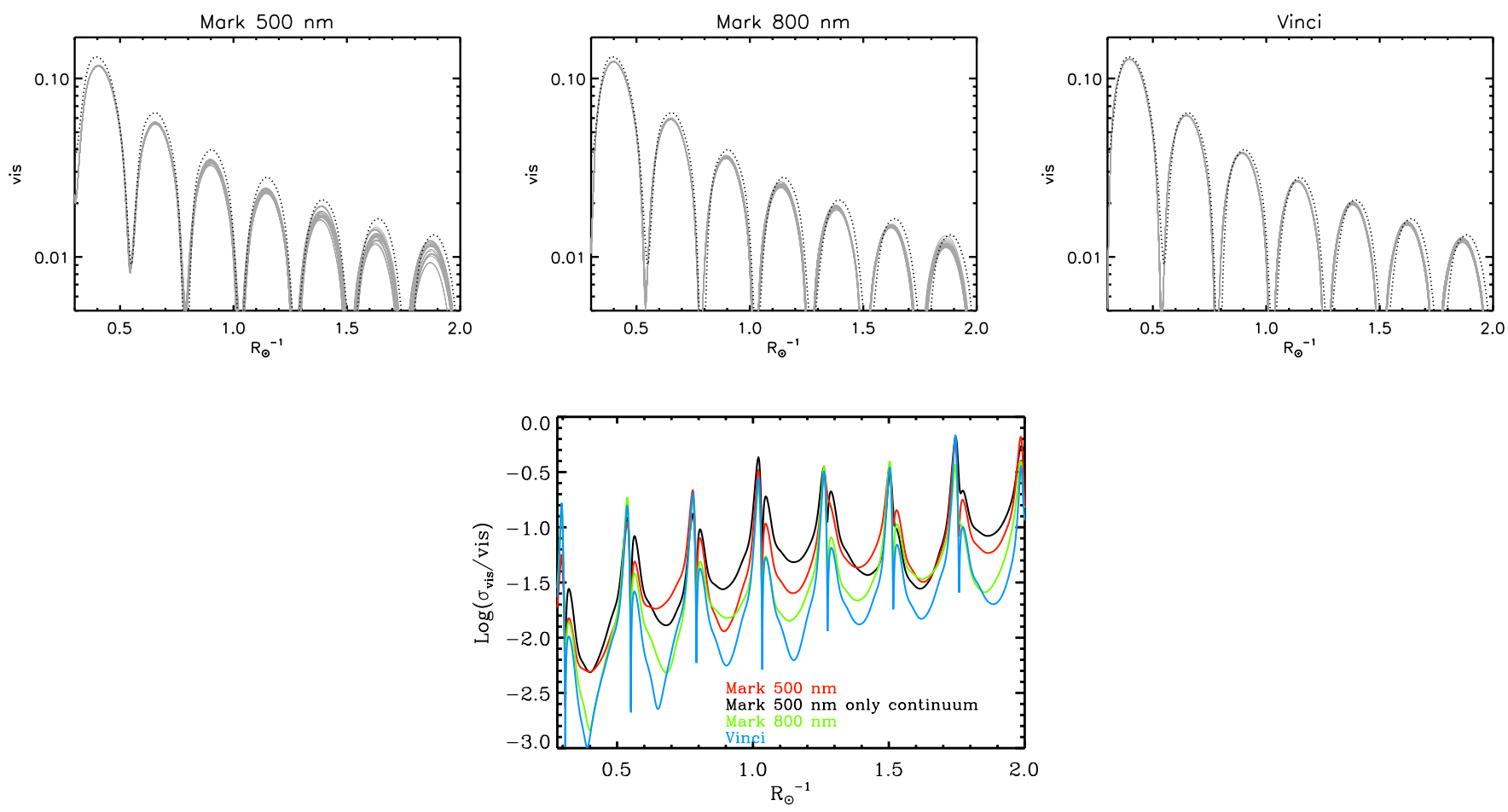

Fig. 6. Top panels: visibility curves (vis) from the Procyon simulation in the filters computed in this work. The visibilities are computed for 36 different azimuth angles $5^{\circ}$ apart (thin gray lines). The dotted line is an uniform disk scaled to match the same radius, i.e. the first null in visibility curve. The visibilities are displayed only longward of the first null visibility point. A logarithmic scale is used on the $y$-axis. Bottom panel: visibility fluctuations $(\sigma / \overline{v i s})$ with respect to the visibility average value $(\overline{v i s})$ as a function of spatial frequencies for all the considered filters.

The first null point of the visibility is mostly sensitive to the radial extension of the observed object (e.g., Quirrenbach 2001; and Chiavassa et al. 2010b, for an application to RHD simulations), while the first null point and the second lobe of the visibility curves are sensitive to the limb-darkening (Hanbury Brown et al. 1974). Since we wish to concentrate on the small-scale structure of the surface, the visibility curves of Fig. 6 are plotted longward of the first null point. They indicate that the fluctuations increase with spatial frequencies owing to deviations from the circular symmetry relative to uniform disk visibility. This dispersion is clearly larger in the optical filter at $500 \mathrm{~nm}$ and appreciable from longward of the top of third lobe. Moreover, it is also noticeable that the synthetic visibilities are systematically lower than for the uniform disk with a weaker divergence for the VINCI filter. This is due to the non-negligible center-to-limb effect visible in Figs. 3 and 4.

The bottom panel of Fig. 6 displays the one $\sigma$ visibility fluctuations, $F$, with respect to the average value $\overline{v i s}$ ( $F=$ $\sigma / \overline{v i s})$. The dispersion increases with spatial frequency owing to the small-scale structure on the model stellar disk (see e.g. Chiavassa et al. 2010b). The dispersion is stronger in the case of the MARK III $500 \mathrm{~nm}$ filter with respect to the redder filters.

Figure 4 shows small differences between the average intensity profiles of the MARK III $500 \mathrm{~nm}$ filter with and without considering the spectral lines in our calculations. Therefore, we computed visibility curves in both cases and found that the visibility fluctuations are indistinguishable (Fig. 6, bottom panel). While the molecular absorption can cause a strong difference in the stellar surface appearance (and consequentially also on the visibility curves) in the case of cool evolved stars (e.g., the contribution of $\mathrm{H}_{2} \mathrm{O}$ to the radius measurement for red supergiants stars, Chiavassa et al. 2010b), this is not the case for
Procyon, where the atomic lines are not strong enough to cause an appreciable effect and the atmosphere is also very compact.

The bottom panel of Fig. 6 also shows that, on the top of the second lobe $\left(\sim 0.4 R_{\odot}^{-1}\right)$, the fluctuations are of about $\sim 0.5 \%$ of the average value for the MARK III $500 \mathrm{~nm}$ filter and $\sim 0.1 \%$ for MarK III $800 \mathrm{~nm}$ and VINCI filters. From the top of the third lobe $\left(\sim 0.6 R_{\odot}^{-1}\right)$ on, the fluctuations are $\sim 2 \%$ of the average value in the optical region, which is larger than the instrumental error of VEGA on CHARA ( 1\%, Mourard et al. 2009).

We note that our method for constructing realizations of stellar disk images inevitably introduces some discontinuities between neighbouring tiles by randomly selecting temporal snapshots and cropping intensity maps at high latitudes and longitudes. However, Chiavassa et al. (2010a) proved that the signal artificially introduced into the visibility curves is largely weaker than the signal due to the inhomogeneities across the stellar surface.

\section{Fundamental parameters}

\subsection{Multiwavelength angular diameter fits in the MARK III and VINCI filters}

We used the data from the MARK III interferometer at 500 and $800 \mathrm{~nm}$ (Mozurkewich et al. 1991) plus additional measurements at $500 \mathrm{~nm}$ reported in Aufdenberg et al. (2005). We note that Mozurkewich et al. (1991) do not provide a calibrator list, even though they claim that the calibrators are all smaller that the science star. The MARK III $500 \mathrm{~nm}$ filter has points on the first and the second lobe, while the MARK III $800 \mathrm{~nm}$ filter has limited baselines in the first lobe. For the MARK III $500 \mathrm{~nm}$ filter, we used only the data for baselines larger than $20 \mathrm{~m}$ because at 
Table 3. Best-fit angular diameters, in mas, for RHD simulation with method (1) $\left(\theta_{\text {Method1 }}\right)$ and method (2) $\left(\theta_{\text {Method2 }}\right)$ described in Sect. 3.1; and for the uniform disk.

\begin{tabular}{lccc}
\hline \hline Filter & $\theta_{\text {Method1 }}$ & $\theta_{\text {Method2 }}$ & $\theta_{\mathrm{UD}}$ \\
\hline MARK 500 nm & $5.313 \pm 0.03$ & $5.324 \pm 0.03$ & $5.012 \pm 0.03$ \\
MARK 800 nm & $5.375 \pm 0.06$ & $5.370 \pm 0.06$ & $5.208 \pm 0.06$ \\
VINCI & $5.382^{a} \pm 0.03$ & $5.397^{a} \pm 0.03$ & $5.326 \pm 0.03$ \\
\hline
\end{tabular}

Notes. The error is one $\sigma$ with respect to the average value. ${ }^{(a)}$ Since the resulting diameters are very similar, we adopted an average angular diameter of 5.390 for all the calculations in the article.

shorter baselines the error bars are $>10 \%$ of the squared visibility. We also used VINcI data from the Very Large Telescope Interferometer (Kervella et al. 2004a; Aufdenberg et al. 2005). The wavelength of these observations is around $2.2 \mu \mathrm{m}$ and the baselines go up to $66 \mathrm{~m}$, but are significantly lower in the first lobe of the Procyon visibility curve.

We used two independent methods to determine the angular diameters:

1. we obtained the synthetic visibilities of Fig. 6 using the spherical tiling method described above and scaled them to absolute model dimensions (using Eqs. (3) and (4)) to match the interferometric observations;

2. we used the method described in Aufdenberg et al. (2005) and Bigot et al. $(2006,2011)$ to compute the normalized fringe visibilities using the van Cittert-Zernike theorem.

Method (1) is based on the Fourier transform of the synthetic disk images (Fig. 3): the visibilities vary for different cuts through the centers of synthetic disk images (i.e., the position of the first null changes its position). Method (2) is based on the integration of the spatial average intensity profile. For both methods, we used a Levenberg-Marquardt least squares minimization.

Table 3 reports the best-fit angular diameters. While for the uniform disk, there is a significantly wide range of values among the different filters, the angular diameters from the RHD simulation are closer and overlap with the uncertainties. The two independent methods used in Table 3 show a clear tendency of the optical diameters to appear smaller than in the infrared $\left(\frac{\theta_{\text {Mark III }}}{\theta_{\text {Vinci }}} \sim 0.99\right)$. The possible explanation of the different radius between MARK III and VINCI are: (i) the RHD model used is not fully appropriate to the observations, for instance the centerto-limb variation may not be correct because, for instance, the points on the second lobe (sensitive to the center-to-limb variation) of MARK $500 \mathrm{~nm}$ filter do not match very well (Fig. 7, top panel); (ii) the data of MARK III may present problems (e.g., calibrator or systematics).

In addition to this, we also conclude that the two methods give consistent results and can be used without distinction to perform angular diameter fits at least for dwarf stars. In the case of cool evolved stars with low surface gravity $(\log g<2.0)$, the surface asymmetry may strongly affected the observed shape of the star and its radius depends on the orientation of the projected baseline (Chiavassa et al. 2009).

The angular diameters found for the VINCI filter (Table 3) are in fairly good agreement with Aufdenberg et al. (2005), who found $5.403 \pm 0.006$ mas. However, $\theta_{\text {vinci }}$ is $\sim 2 \%$ smaller than: (i) $5.448 \pm 0.03$ mas (Kervella et al. 2004b), based on the fit of the VINCI with baseline points of Fig. 7 lower than $22 \mathrm{~m}$; (ii) $5.48 \pm$ 0.05 mas (Allende Prieto et al. 2002), obtained with Eq. (7) from

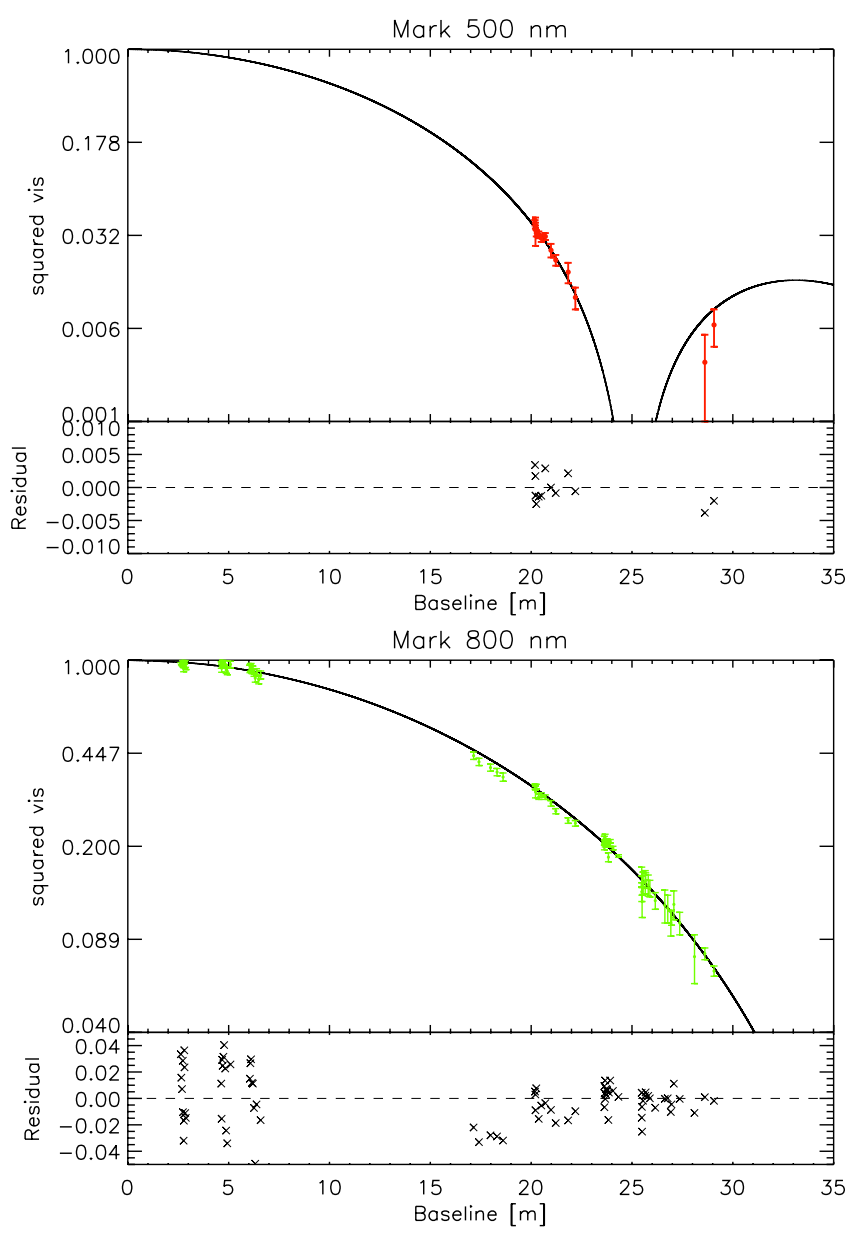

Vinci

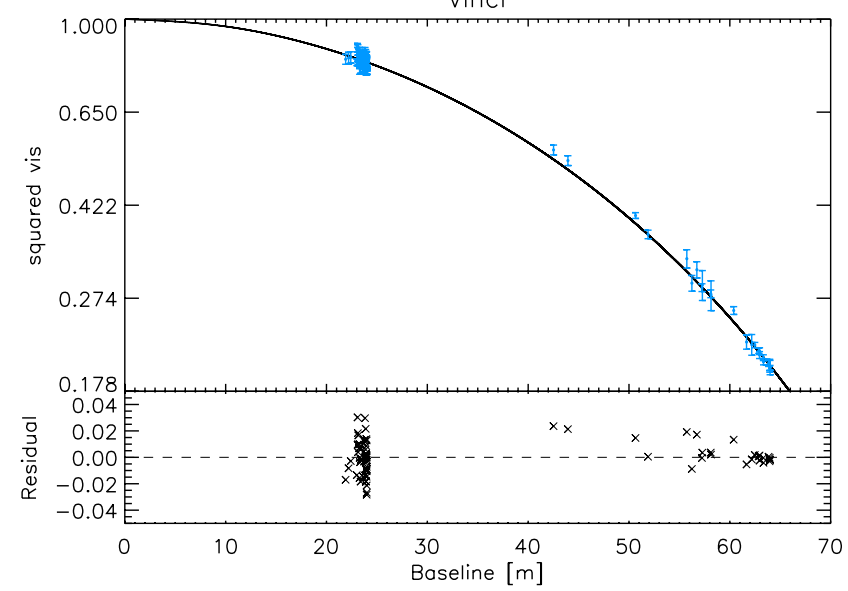

Fig. 7. Best matching synthetic visibility from Fig. 6 for the MARK 500, MARK 800, and VINCI data. The stellar disk maps in each filter have been scaled to match the radius of the star. For the Mark $500 \mathrm{~nm}$ filter, we used only the data with baselines larger than $20 \mathrm{~m}$.

the linear radius of $2.071 \pm 0.020 R_{\odot}$; and (iii) $5.50 \pm 0.17$ mas (Mozurkewich et al. 1991). Our $\theta_{\text {Vinci }}$ value is also close to the value $5.326 \pm 0.068$ mas found by Casagrande et al. (2010) with the infrared flux method.

Transforming the VINCI angular diameter to a linear radius (Eq. (7)), we found $2.019 R_{\odot}$. This value is compared to what we chose as a reference in Table 1: $2.055 R_{\odot}$. The difference is $0.023 R_{\odot}$, which is $16 \mathrm{Mm}$ (i.e., 0.7 times the numerical box of RHD simulation, $22 \mathrm{mM}$, Table 1 , fourth column). We checked 
that this has a negligible impact on the observables found in this work using the spherical tiling method described in Sect. 2.2.

The astrometric mass $\left(M=1.430 \pm 0.034 M_{\odot}\right.$ Gatewood \& Han 2006, used in Sect. 3.3) combined with our interferometric diameter leads to a new gravity $\log g=4.01 \pm 0.03\left[\mathrm{~cm} / \mathrm{s}^{2}\right]$, which is higher by 0.05 dex than the value derived in Allende Prieto et al. (2002). The contribution of the revised Hipparcos parallax to the $\log g$ change is $\sim 0.01$ dex.

\subsection{The effective temperature}

The effective temperature is determined from the bolometric flux $F_{\text {BOL }}$ and the angular diameter $\theta$ in the VINCI filter (average between the two methods in Table 3, $\theta=5.390$ mas) by the relation

$T_{\mathrm{eff}}=\left(\frac{4 F_{\mathrm{BOL}}}{\sigma_{\mathrm{st}} \theta^{2}}\right)^{0.25}$,

where $\sigma_{\text {st }}$ stands for the Stefan-Boltzmann constant. Our derived diameter is smaller by $\sim 2 \%$ than those of Mozurkewich et al. (1991) or Kervella et al. (2004b), hence our derived effective temperature has to be higher by $\sim 1 \%$ than those of Allende Prieto et al. (2002) or Aufdenberg et al. (2005), who used the afore mentioned references of Mozurkewich et al. and Kervella et al. There are several sources of bolometric flux leading to slightly different $T_{\text {eff }}$. The values of $F_{\mathrm{BOL}}=(18.20 \pm$ 0.43 ) $10^{-6} \mathrm{erg} \mathrm{cm}^{-2} \mathrm{~s}^{-1}$ (Fuhrmann et al. 1997) and $F_{\mathrm{BOL}}=$ (17.82 \pm 0.89 ) $10^{-6} \mathrm{erg} \mathrm{cm}^{-2} \mathrm{~s}^{-1}$ (Aufdenberg et al. 2005) imply $T_{\text {eff }, 3 \mathrm{D}}=6591 \pm 43 \mathrm{~K}$ and $6556 \pm 84 \mathrm{~K}$, respectively.

Our new 3D $T_{\text {eff,3D }}$ returns a value closer to the value $T_{\text {eff,IR }}=6621 \pm 80 \mathrm{~K}$ obtained by Casagrande et al. (2010, and Casagrande, priv. comm.) than the old derived value of $6516 \pm 87 \mathrm{~K}$ (Aufdenberg et al. 2005).

The influence of the uncertainties in the selected fundamental parameters $\left(T_{\mathrm{eff}}, \log g,[\mathrm{Fe} / \mathrm{H}]\right)$ on our RHD model atmosphere has a negligible impact on the limb-darkening and therefore on the derived angular diameter and $T_{\text {eff }}$. This has been tested for HD 49933 (Bigot et al. 2011), a star similar to Procyon.

\subsection{Independent asteroseismic determination of the radius}

The radius of the star can be derived from its oscillation spectrum. The frequency $v_{\max }$ of the maximum in the power spectrum is generally assumed to scale with the acoustic cut-off frequency of the star (e.g. Brown et al. 1991; Kjeldsen \& Bedding 2011), hence $v_{\max } \approx g / \sqrt{T_{\text {eff }}}$. It is then straightforward to derive the radius

$\frac{R}{R_{\odot}}=\left(\frac{M}{M_{\odot}} \frac{v_{\max , \odot}}{v_{\max }}\right)^{0.5}\left(\frac{T_{\mathrm{eff}}}{T_{\mathrm{eff}, \odot}}\right)^{-0.25}$.

The validity of this scaling relation has been verified by large asteroseismic surveys (e.g. Bedding \& Kjeldsen 2003; Verner et al. 2011). The value of $v_{\max }$ derived from photometry is accurately determined, $1014 \pm 10 \mu \mathrm{Hz}$ (Arentoft et al. 2008). The solar value of $v_{\max }$ is taken from Belkacem et al. (2011). We emphasize that the dependence on $T_{\text {eff }}$ in Eq. (6) is weak, hence the derived radius is very insensitive to the selected value of the effective temperature. We use the value of $T_{\text {eff }}=6591 \pm 43 \mathrm{~K}$ derived in Sect. 3.2, since it is closer to the infrared flux method determination. Since Procyon is a binary star, the mass can by determined from the astrometric orbital elements and the third Kepler's law. However, the derived value remains the subject of debate.

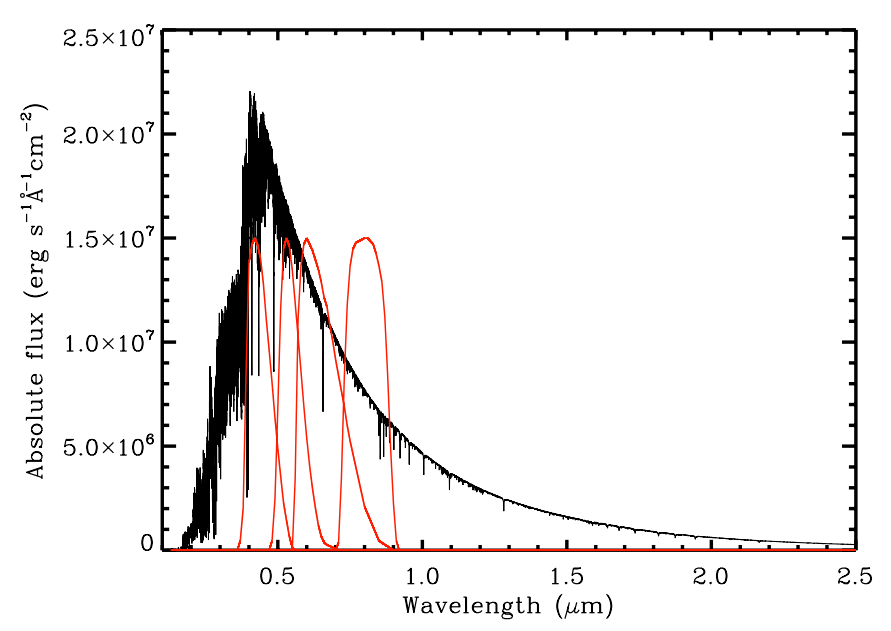

Fig. 8. Spatially and temporally average synthetic SED from the UV to the near infrared. Red curves correspond to Bessell (1990b) for the filters $B V R I$

Table 4. Photometric colors for the RHD simulation of Table 1 and the corresponding observations of Bessel (1990a).

\begin{tabular}{lcccc}
\hline \hline & $B-V$ & $V-R$ & $R-I$ & $V-I$ \\
\hline RHD simulation & 0.419 & 0.255 & 0.252 & 0.507 \\
Observation & 0.420 & 0.245 & 0.245 & 0.490 \\
\hline
\end{tabular}

Girard et al. (2000) found a mass of $M=1.497 \pm 0.037 M_{\odot}$, whereas Gatewood \& Han (2006) found $M=1.430 \pm 0.034 M_{\odot}$. As discussed in the introduction, we prefer to keep the value of Gatewood \& Han since stellar evolution models that use this mass, agree with the age of the white dwarf companion. Using these values, we found a radius of $R=2.023 \pm 0.026 R_{\odot}$. We can translate this radius into an angular diameter using the relation

$\theta_{\text {seismic }}=2\left(R / R_{\odot}\right) \pi_{p} \tan \left(\theta_{\odot} / 2\right)=5.36 \pm 0.07 \mathrm{mas}$,

with the solar angular radius $\theta_{\odot} / 2=959.64 \pm 0.02$ arcsecs (Chollet \& Sinceac 1999) and the parallax $\pi_{p}=284.56 \pm$ 1.26 mas (van Leeuwen 2007). This radius agrees well with our interferometric value within error bars.

\section{Spectrophotometry and photometry}

The radiative transfer code OpтIм3D includes all the up-to-date molecular and atomic line opacities. This allows for very realistic spectral synthetic RHD simulations for wavelengths from the ultraviolet to the far infrared. It is then possible to compute realistic synthetic colors and compare them to the observations. Figure 8 shows the SED computed along rays of four $\mu$-angles $[0.88,0.65,0.55,0.34]$ and four $\phi$-angles $\left[0^{\circ}, 90^{\circ}, 180^{\circ}, 270^{\circ}\right]$, and a temporal average of all selected snapshots (as in Fig. 2). The shape of SED reflects the mean thermal gradient of the simulations.

We used the prescriptions of Bessell (1990b) to compute the color indices for the filters $B V R I$. Table 4 compares the synthetic colors with the observed ones. The difference is very small.

We compared the absolute spectrophotometry measurements at ultraviolet and visual wavelengths collected by Aufdenberg et al. (2005) with the synthetic SED. The data come from: (i) the Goddard High Resolution Spectrograph (GHRS) data sets Z2VS0105P (PI A. Boesgaard), Z17X020CT, Z17X020AT, Z17X0208T (PI J. Linsky) from 136 

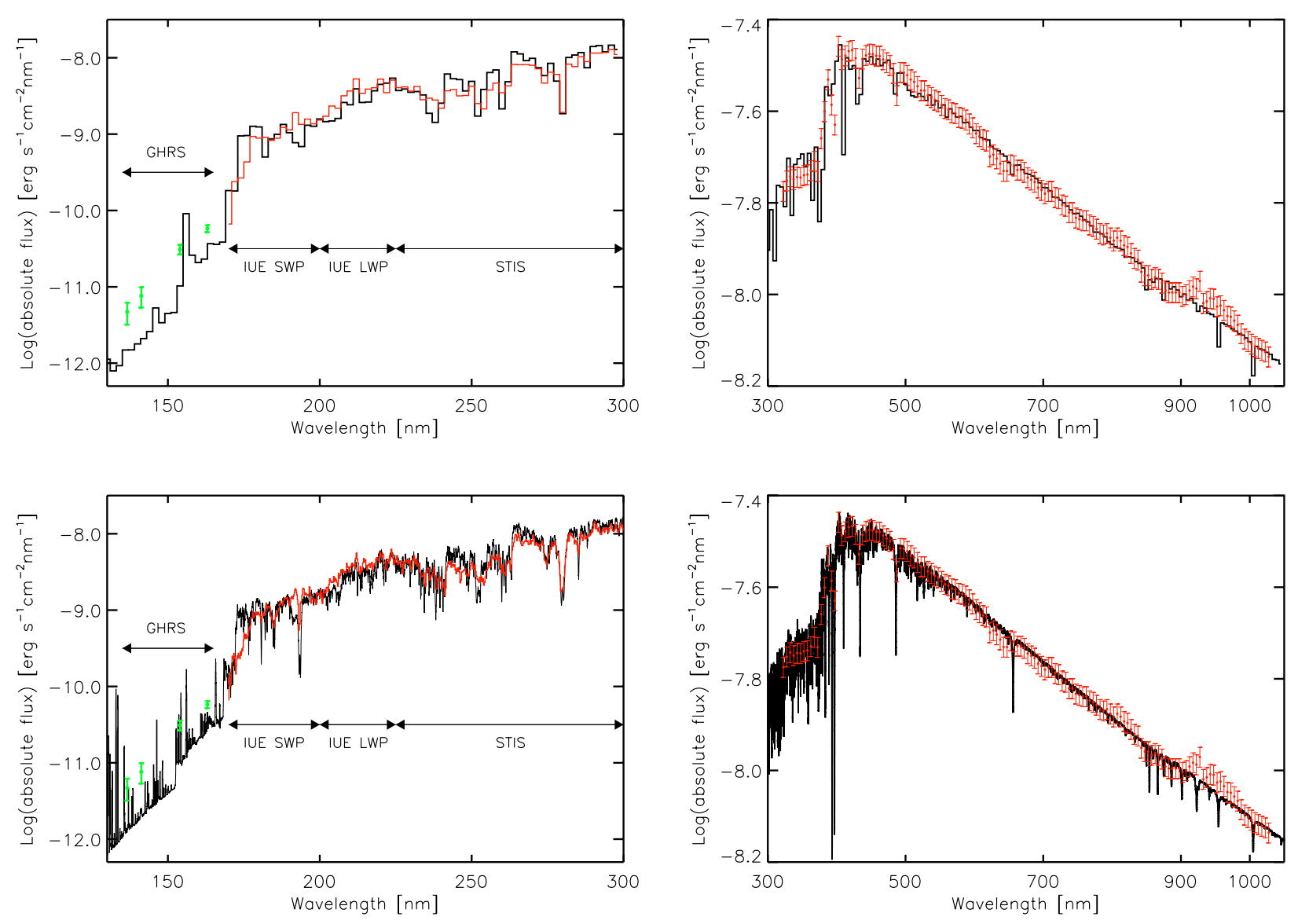

Fig. 9. Comparisons between synthetic SED (black) and spectrophotometric measurements at ultraviolet and visual wavelengths (green dots with error bars in left column plots and red line). Top row: the models and data are binned to $2 \mathrm{~nm}$ resolution in the UV for clarity and to $5 \mathrm{~nm}$ in the visual to match the resolution of the observed spectrophotometry of Glushneva et al. (1992). Bottom row: same as above with the synthetic SED and the observations at higher spectral resolution. The synthetic SEDs are scaled in absolute flux using an angular diameter of 5.390 mas found from the fit of VINCI filter observations.

and $160 \mathrm{~nm}$; (ii) the International Ultraviolet Explorer (IUE) Rodríguez-Pascual et al. (1999) from 170 to $306 \mathrm{~nm}$; (iii) the Hubble Space Telescope imaging Spectrograph (STIS) from 220 to $410 \mathrm{~nm}$; and (iv) the visual and near-infrared wavelength data of Glushneva et al. (1992). The data from the GHRS are of far higher quality that any other measurements below $160 \mathrm{~nm}$ because the continuum declines by more than a factor of 100 here, too much for the limited dynamic range of IUE. The flux was estimated by computing the mean flux between the emission lines in each spectrum incorporating the flux uncertainties provided with each calibrated data set (Aufdenberg et al. 2005).

Figure 9 (top row) shows that our synthetic SED matches fairly well the observations from the UV to the near-IR region. This result can be compared to the already good agreement found with the composite RHD model computed using the $\mathrm{CO}^{5} \mathrm{BOLD}$ code (Aufdenberg et al. 2005). Moreover, the bottom row of the figure shows that at higher spectral resolution, the agreement is even more remarkable. The RHD model also match the observations between 136 and $160 \mathrm{~nm}$, which are supposed to form at depths beneath its chromosphere (Aufdenberg et al. 2005) and are thus being affected by the convection-related surface structures. We note that the ultraviolet SED longward of $160 \mathrm{~nm}$ may be affected by the non-LTE treatment of irongroup elements. Short \& Hauschildt (2005) found that nonLTE models for the Sun have up to $20 \%$ more near-UV flux than LTE models. It is impossible to determine whether this difference is also present in Procyon because either the RHD model calculation and the post-processing calculations were performed for a LTE approximation. The ultraviolet SED may also be affected by scattering at these wavelengths. This effect is also not included in our calculations. However, Hayek et al. (2010) demonstrated that, in RHD simulations, the scattering does not have a significant impact on the photospheric temperature structure in the line forming region for a main sequence star.

We conclude that the mean thermal gradient of the simulation, reflected by the SED closely agrees with that of Procyon.

\section{Closure phases and perspectives for hot Jupiter detection}

Interferometry can permit the direct detection and characterization of extrasolar planets. It has been claimed that differential interferometry could be used to obtain spectroscopic information, and determine both the planetary mass, and the orbit inclination of extrasolar planets around nearby stars (Segransan et al. 2000; Lopez et al. 2000; Joergens \& Quirrenbach 2004; Renard et al. 2008; Zhao et al. 2008; van Belle 2008; Matter et al. 2010; Absil et al. 2011; Zhao et al. 2011). However, current interferometers lack sufficient accuracy for such a detection. When observing a star with a faint companion, their fringe patterns add up incoherently and the presence of a planet causes a slight change in 
the phases and, consequently, the closure phases. This difference can be measured with a temporal survey and should be corrected for the intrinsic closure phases of the host star.

In this framework, theoretical predictions of the closure phases of the host stars are crucial. The closure phase between three (or more) telescopes is the sum of all phase differences, and its evaluation enables us to remove the atmospheric contribution, leaving the phase information of the object visibility unaltered. The major biases or systematic errors in the closure phases come from non-closed triangles introduced in the measurement process, which in principle, can be precisely calibrated. Therefore, the closure phase is a good observable for stable and precise measurements (e.g. Monnier 2007), providing an important complementary piece of information, and helping to reveal asymmetries and inhomogeneities across stellar disk images.

\subsection{Closure phases from the star alone}

Figure 10 displays deviations from the axisymmetric case (zero or $\pm \pi$ ) that occur particularly in optical filters. There is a correlation between Figs. 10 and 6 because the scatter in the closure phases increases with spatial frequencies in the same way as the visibilities: smaller structures need large baselines to be resolved. Moreover, it is clear that the closure phase signal becomes important for frequencies higher than $\sim 0.6 R_{\odot}^{-1}$ (top of the third visibility lobe, i.e., vis $\sim 0.06$ from Fig. 6). These predictions can be constrained by the level of asymmetry and inhomogeneity of stellar disks by accumulating observations on closure phase at short and long baselines.

Observing dwarf stars at high spatial resolution is thus crucial to characterize the granulation pattern using closure phases. This requires observations at high spatial frequencies (from the third lobe on) and especially in the optical range. Since the maximum baseline of CHARA is $331 \mathrm{~m}$ (ten Brummelaar et al. 2005) for a star like Procyon with a parallax of 284.56 mas (van Leeuwen 2007), a baseline of 55 (240) meters is necessary to probe the third lobe at $0.5(2.2) \mu \mathrm{m}$. For comparison, the nominal measurement error for the CHARA array is $0.3^{\circ}$, and the errors peak at a performance of $0.1^{\circ}$ for a shorter triangle (Zhao et al. 2008, to be compared with closure phases value of Fig. 10).

\subsection{Closure phases for the hosting star and the hot Jupiter companion}

Among the detected exoplanets, the direct detection and the characterization of their atmospheres currently appears to be within reach for a very close planet-star system $(<0.1 \mathrm{AU})$ and planets with temperatures $\gtrsim 1000 \mathrm{~K}$, implying that their infrared flux is $\sim 10^{-3}$ of their host stars. Since the bulk of the energy from hot Jupiters emerges from the near-infrared across the range 1-3 $\mu \mathrm{m}$ (Burrows et al. 2008), interferometry in the near-infrared band (as in the range of the VINCI filter centered on $\sim 2.2 \mu \mathrm{m}$ ) can provide measurements that permit us to detect and characterize the planets. The detection of hot Jupiters from Earth is a remarkable endeavor because it is challenging and at the limits of the current performances of interferometry, and even when these are reached the signal from the host stars must also be reliable detected. As can be clearly seen in the synthetic stellar disk images of Fig. 3, the granulation is a non-negligible aspect of the surface of dwarf stars and has an a significant effect on the closure phases (Fig. 10).
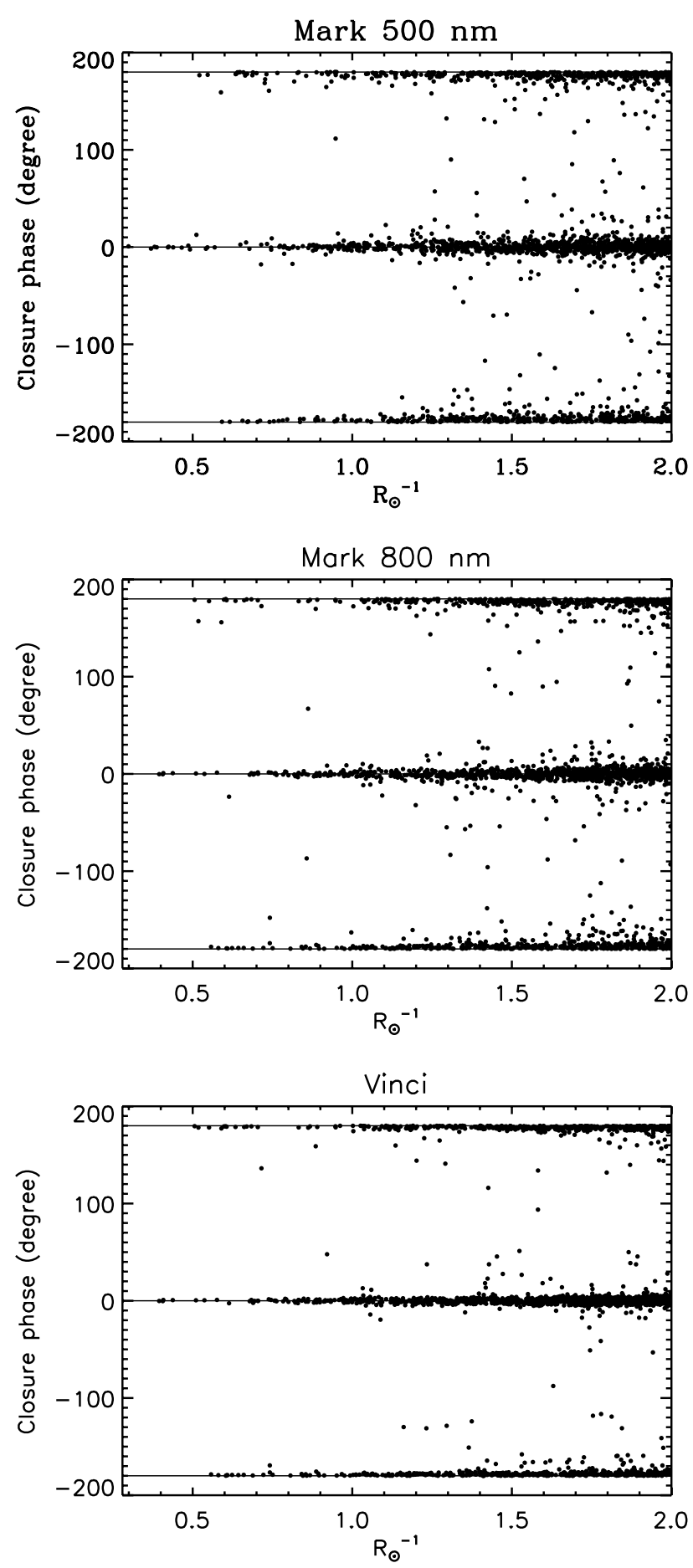

Fig. 10. Scatter plot of closure phases of 2000 random baseline triangles with a maximum linear extension of $\sim 155 \mathrm{~m}$ at $500 \mathrm{~nm}, \sim 250 \mathrm{~m}$ at $800 \mathrm{~nm}$, and $\sim 680 \mathrm{~m}$ at $2.2 \mu \mathrm{m}$, respectively. A parallax of 284.56 mas (van Leeuwen 2007) and an apparent radius of 5.390 mas have been used.

To estimate the impact of the granulation noise on the planet detection, we used the RHD simulation of Procyon and added a virtual companion to the star. The modeling of the flux of an irradiated planet requires the careful adherence to the radiative transfer conditions related to the stellar irradiation and therefore we used the models of Barman et al. (2001). In particular, we used the spectra of a hot irradiated extrasolar planet around a star with about the same spectral type as Procyon, a mass of 1 Jupiter 


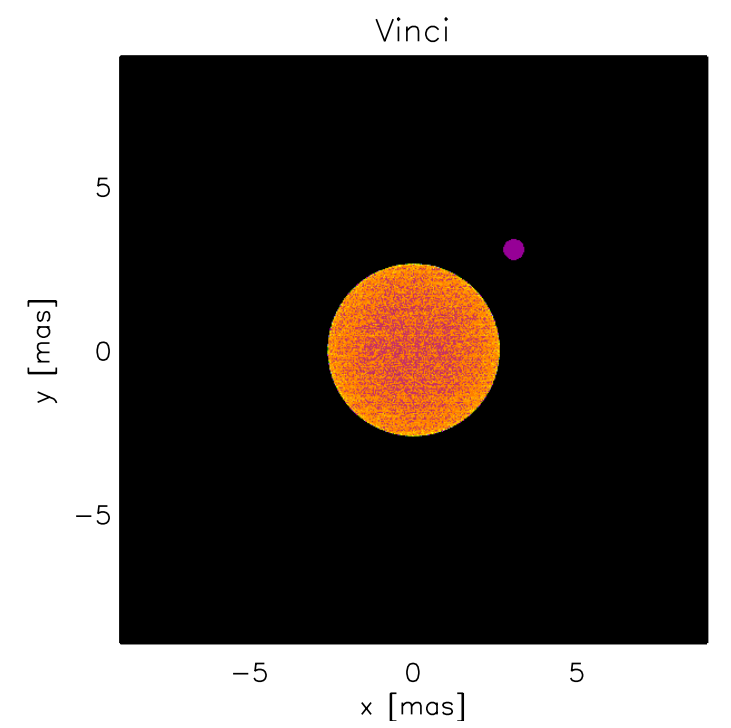

Fig. 11. Synthetic stellar disk images (yellow-orange scale) of the simulation in VINCI filter (the intensity range is [0.3-0.9 $\times$ $\left.10^{5}\right] \mathrm{erg} \mathrm{cm}^{-2} \mathrm{~s}^{-1} \AA^{-1}$ ), together with a hot Jupiter (pink) at a distance of 0.1 AU for a star with a parallax of 284.56 mas (van Leeuwen 2007). The intensity of the hot Jupiter is $\sim 11$ times lower than the maximum stellar surface intensity.

mass, and an intrinsic temperature $1000 \mathrm{~K}$. We assumed a radius of 1.2 Jupiter radii and various orbital distances [0.1, 0.25, 0.5, 1.0] AU following Matter et al. (2010). The atmospheric composition of the hot Jupiter is identical to the two models of Allard et al. (2001) where: (1) dust (particles and grains) remains in the upper atmosphere and (2) dust has been removed from the upper atmosphere by condensation and gravitational settling.

Figure 11 shows the geometrical configuration of the starplanet system for a particular distance. We first averaged the exoplanetary spectrum in the range of the MARK III $800 \mathrm{~nm}$ and VINCI filters (we had no data for the exoplanet spectrum in the range of MARK III $500 \mathrm{~nm}$ filter), and then used this intensity for the stellar companion in Fig. 11. The intensity of the planet is stronger in the infrared than the optical. The ratio of the stellar intensity at its center (i.e., $\mu=1$ ), $I_{\text {Vinci }}$ or $I_{\text {Mark800, to the }}$ planetary integrated intensity in the same filter, $I_{\text {planet }}$, is

$\frac{I_{\text {Vinci }}}{I_{\text {planet }}}=11.5,31.7,42.7,46.3$,

$\frac{I_{\text {Mark800 }}}{I_{\text {planet }}}=34.7,104.0,130.0,182.5$

for distances $[0.1,0.25,0.5,1.0] \mathrm{AU}$, respectively.

We computed the closure phases from the image of Fig. 11 and for similar systems corresponding to the VINCI and MARK III $800 \mathrm{~nm}$ filters and the distances reported above. These closure phases were compared with the resulting phases computed for exactly the same triangles but for a system without a planet. Figure 12 displays the absolute differences between closure phases with and without a hot Jupiter. Setting as a reference the closure phase nominal error of CHARA $\left(0.3^{\circ}\right)$ and also $1^{\circ}$ (horizontal lines in the plot), only the VINCI filter leads to differences that should be detectable on the third lobe $\left(0.5 \lesssim R_{\odot}^{-1} \lesssim 0.8\right)$, while there is no signature on the second lobe $\left(R_{\odot}^{-1} \lesssim 0.5\right)$. The absolute difference increases as a function of spatial frequency, and is indistinguishable for all the planet' distances for frequencies higher than $0.8 \sim R_{\odot}^{-1}$.
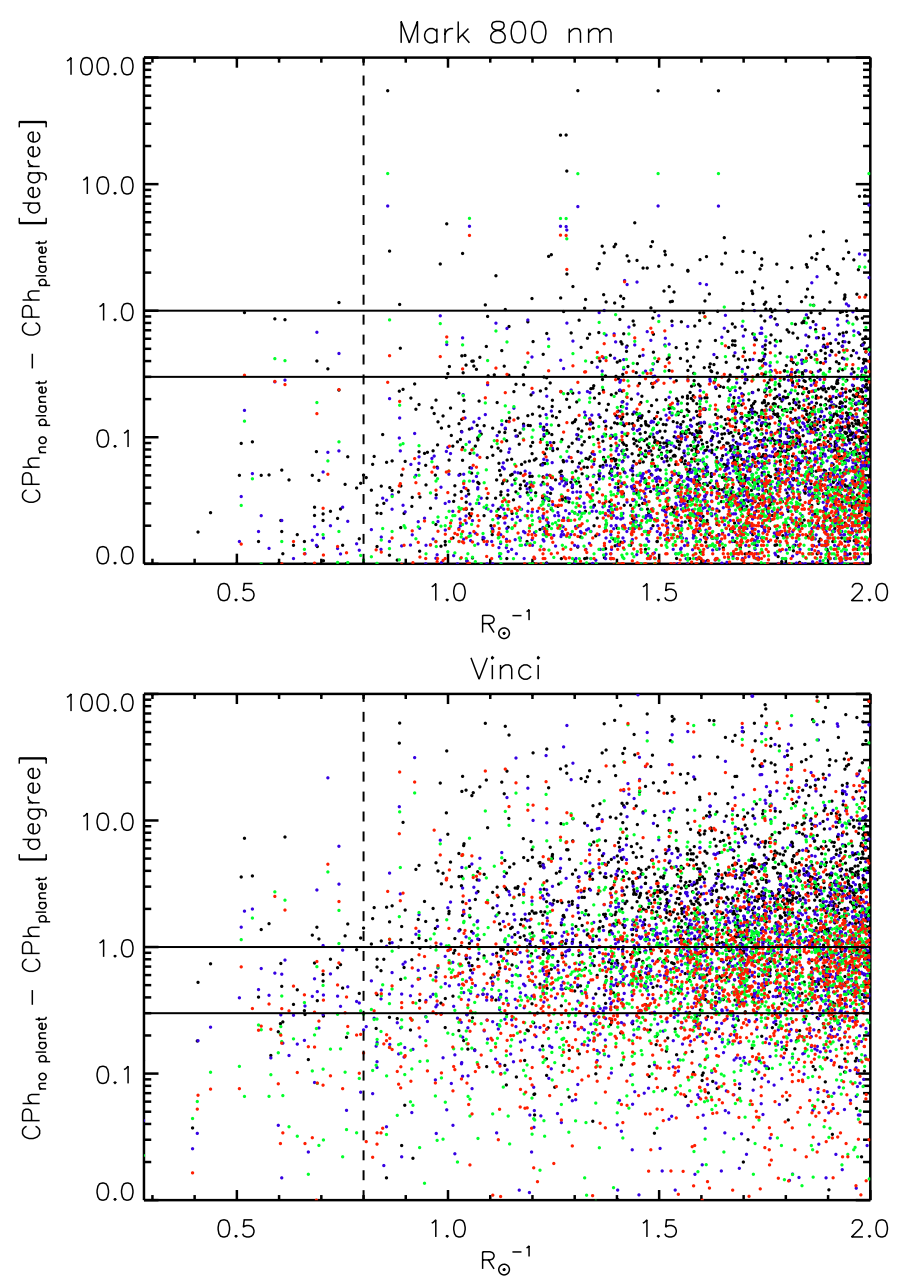

Fig. 12. Absolute differences between the closure phases computed for the 2000 random baseline triangles in the case of a system without the presence of a hot Jupiter companion $\left(C P h_{\text {noplanet }}\right)$ and a system starplanet $\left(C P h_{\text {planet }}\right.$, see text and Fig. 11). The horizontal solid lines indicate the 1.0 and 0.3 degrees and the vertical dashed line the spatial frequency corresponding to the end of the third visibility lobe. The black, blue, green, and red colors denote the planet-star distance of $[0.1,0.25$, $0.5,1.0] \mathrm{AU}$, respectively. The maximum linear extension, the apparent radius, and the parallax are the same as Fig. 10.

The purpose of this section has been to illustrate the change in the signal due to the granulation noise in the detection of a hot Jupiter around Procyon-like stars using closure phases. To date, no companions have been detected around Procyon except for a white dwarf astrometric companion detected previously in the 19 th century (see Sect. 1).

Future studies of hot Jupiter atmospheres will reveal their composition, structure, dynamics, and planet formation processes, and for these it will be very important to have a complete knowledge of the host star to reach these aims.

\section{Conclusions}

We have provided new predictions of interferometric and spectroscopic observables for Procyon, based on a RHD hydrodynamical simulation, that affect the fundamental parameter determination of the star and are important for the detection of hot Jupiter exoplanets.

We have studied the effect of the granulation pattern on the center-to-limb intensity profiles and provided limb-darkening 
coefficients in the optical as well as in the infrared. We have demonstrated that the synthetic visibility curves produced by the RHD simulation are systematically lower than those for a uniform disk and that this effect is stronger in the optical filters. In addition to this, visibilities display fluctuations that increase with spatial frequencies (i.e., departure from circular symmetry) that becomes $\sim 2 \%$ on the top of the third lobe in MARK III $500 \mathrm{~nm}$ filter. However, in the MARK III $800 \mathrm{~nm}$ and VINCI filter the dispersion is much weaker.

We have derived new angular diameters at different wavelengths using two independent methods based on the RHD simulation. The angular diameter in the VINCI filter is $\theta=5.390$ mas, which leads to an effective temperature of $T_{\text {eff }}=6591 \pm 43 \mathrm{~K}$ or $6556 \pm 84 \mathrm{~K}$, depending on the bolometric flux considered. This value is now more consistent with $T_{\text {eff }, \text { IR }}=6621 \pm 80 \mathrm{~K}$ from the infrared flux method (Casagrande et al. 2010, and Casagrande, priv. comm.).

Using an independent estimate of the radius from asteroseismology, we found that $\theta_{\text {seismic }}=5.36 \pm 0.07$ mas. This radius agrees well with our interferometric value within the error bars.

A combination of the astrometric mass and our new interferometric diameter provides a new gravity $\log g=4.01 \pm$ $0.03\left[\mathrm{~cm} / \mathrm{s}^{2}\right]$, which is larger by 0.05 dex than the value derived in Allende Prieto et al. (2002).

We accurately compared a synthetic spectrum from a RHD simulation with observations from the ultraviolet to the infrared. The photometric BVRI colors are very similar to the observations, while it is difficult to derive any conclusions for the infrared colors because of the saturation of the observations. In addition, our comparison with the absolute spectrophotometric measurements, collected in Aufdenberg et al. (2005), illustrated close agreement. We concluded that the mean thermal gradient in the simulation, inferred by the SED, is in very good agreement with that of Procyon.

The convection-related surface structures also affected the signal of the closure phases, which show departures from symmetry for about the same spatial frequencies as the visibility curves. We concluded that closure phases that are not equal to either 0 or $\pm \pi$ may be detected with today interferometers such as CHARA in the visible filters where the baselines are long enough to identify the second/third lobes.

We estimated the impact of the granulation noise on the hot Jupiter detection using closure phases around stars with the same spectral type as Procyon. We used the synthetic stellar disks obtained from RHD in the infrared and optical filters and added a virtual companion to the star based on real integrated spectra of irradiated extrasolar planet. We then computed the closure phases for both planet-star and star only systems and found that there is a non-negligible and detectable contamination of the signal of the hot Jupiter by the granulation for spatial frequencies longward of the third lobe. This is valid only in the infrared, where the brightnesses of the hot Jupiters are higher. It is thus very important to have a comprehensive knowledge of the host star to detect and characterize hot Jupiters, and RHD simulations are very important to achieve this aim. In a forthcoming paper, we will extend this analysis to solar-type stars and $\mathrm{K}$ giants across the HR diagram.

Acknowledgements. The authors gratefully thank J. Aufdenberg for his help and enlightening discussions. A.C. is supported in part by an Action de recherche concertée (ARC) grant from the Direction générale de l'Enseignement non obligatoire et de la Recherche scientifique - Direction de la Recherche scientifique - Communauté française de Belgique. A.C. is also supported by the F.R.S.FNRS FRFC grant 2.4513.11. We thank the Rechenzentrum Garching (RZG) for providing the computational resources necessary for this work. This research received the support of PHASE, the high angular resolution partnership between ONERA, Observatoire de Paris, CNRS and University Denis Diderot Paris 7.

\section{References}

Absil, O., Le Bouquin, J.-B., Berger, J.-P., et al. 2011, A\&A, 535, A68 Allard, F., Hauschildt, P. H., Alexander, D. R., Tamanai, A., \& Schweitzer, A. 2001, ApJ, 556, 357

Allende Prieto, C., Asplund, M., García López, R. J., \& Lambert, D. L. 2002, ApJ, 567, 544

Arentoft, T., Kjeldsen, H., Bedding, T. R., et al. 2008, ApJ, 687, 1180

Asplund, M., Grevesse, N., Sauval, A. J., \& Scott, P. 2009, ARA\&A, 47, 481

Atroshchenko, I. N., Gadun, A. S., \& Kostik, R. I. 1989, in Solar and Stellar

Granulation, ed. R. J. Rutten, \& G. Severino, NATO ASIC Proc., 263, 521

Aufdenberg, J. P., Ludwig, H.-G., \& Kervella, P. 2005, ApJ, 633, 424

Auwers, G. F. J. A. 1862, De motu proprio Procyonis variabili

Barban, C., Michel, E., Martic, M., et al. 1999, A\&A, 350, 617

Barman, T. S., Hauschildt, P. H., \& Allard, F. 2001, ApJ, 556, 885

Bedding, T. R., \& Kjeldsen, H. 2003, PASA, 20, 203

Bedding, T. R., Kjeldsen, H., Campante, T. L., et al. 2010, ApJ, 713, 935

Belkacem, K., Goupil, M. J., Dupret, M. A., et al. 2011, A\&A, 530, A142

Bessel, F. W. 1844, MNRAS, 6, 136

Bessel, M. S. 1990a, A\&AS, 83, 357

Bessell, M. S. 1990b, PASP, 102, 1181

Bigot, L., Kervella, P., Thévenin, F., \& Ségransan, D. 2006, A\&A, 446, 635

Bigot, L., Mourard, D., Berio, P., et al. 2011, A\&A, 534, L3

Bonanno, A., Küker, M., \& Paternò, L. 2007, A\&A, 462, 1031

Brown, T. M., Gilliland, R. L., Noyes, R. W., \& Ramsey, L. W. 1991, ApJ, 368, 599

Burrows, A., Budaj, J., \& Hubeny, I. 2008, ApJ, 678, 1436

Casagrande, L., Ramírez, I., Meléndez, J., Bessell, M., \& Asplund, M. 2010, A\&A, 512, A54

Chiavassa, A., Plez, B., Josselin, E., \& Freytag, B. 2009, A\&A, 506, 1351

Chiavassa, A., Collet, R., Casagrande, L., \& Asplund, M. 2010a, A\&A, 524, A93

Chiavassa, A., Haubois, X., Young, J. S., et al. 2010b, A\&A, 515, A12

Chollet, F., \& Sinceac, V. 1999, A\&AS, 139, 219

Code, A. D., Bless, R. C., Davis, J., \& Brown, R. H. 1976, ApJ, 203, 417

Collet, R., Magic, Z., \& Asplund, M. 2011, J. Phys. Conf. Ser., 328, 012003

di Mauro, M. P., \& Christensen-Dalsgaard, J. 2001, in Recent Insights into the

Physics of the Sun and Heliosphere: Highlights from SOHO and Other Space

Missions, ed. P. Brekke, B. Fleck, \& J. B. Gurman, IAU Symp., 203, 94

Dravins, D. 1987, A\&A, 172, 211

Eggen, O. J., \& Greenstein, J. L. 1965, ApJ, 141, 83

Eggenberger, P., Carrier, F., Bouchy, F., \& Blecha, A. 2004, A\&A, 422, 247

Eggenberger, P., Carrier, F., \& Bouchy, F. 2005, New Astron., 10, 195

Freytag, B., Steffen, M., \& Dorch, B. 2002, Astron. Nachr., 323, 213

Freytag, B., Steffen, M., Ludwig, H.-G., et al. 2012, J. Comput. Phys., 231, 919

Fuhrmann, K., Pfeiffer, M., Frank, C., Reetz, J., \& Gehren, T. 1997, A\&A, 323, 909

Gatewood, G., \& Han, I. 2006, AJ, 131, 1015

Gelly, B., Grec, G., \& Fossat, E. 1986, A\&A, 164, 383

Gelly, B., Grec, G., \& Fossat, E. 1988, in Advances in Helio- and Asteroseismology, ed. J. Christensen-Dalsgaard, \& S. Frandsen, IAU Symp., 123,249

Girard, T. M., Wu, H., Lee, J. T., et al. 2000, AJ, 119, 2428

Glushneva, I. N., Kharitonov, A. V., Kniazeva, L. N., \& Shenavrin, V. I. 1992, A\&AS, 92, 1

Gray, D. F. 1967, ApJ, 149, 317

Gray, D. F. 1981, ApJ, 251, 583

Griffin, R. 1971, MNRAS, 155, 139

Guenther, D. B., \& Demarque, P. 1993, ApJ, 405, 298

Guenther, D. B., Kallinger, T., Gruberbauer, M., et al. 2008, ApJ, 687, 1448

Gustafsson, B., Edvardsson, B., Eriksson, K., et al. 2008, A\&A, 486, 951

Hanbury Brown, R., Davis, J., Allen, L. R., \& Rome, J. M. 1967, MNRAS, 137, 393

Hanbury Brown, R., Davis, J., Lake, R. J. W., \& Thompson, R. J. 1974, MNRAS, 167,475

Hartmann, L., Garrison, Jr., L. M., \& Katz, A. 1975, ApJ, 199, 127

Hayek, W., Asplund, M., Carlsson, M., et al. 2010, A\&A, 517, A49

Joergens, V., \& Quirrenbach, A. 2004, SPIE Proc. 5491, ed. W. A. Traub, 551

Kervella, P., Gitton, P. B., Segransan, D., et al. 2003a, in SPIE Proc. 4838, ed. W. A. Traub, 858

Kervella, P., Thévenin, F., Morel, P., Bordé, P., \& Di Folco, E. 2003b, A\&A, 408, 681

Kervella, P., Thévenin, F., Ségransan, D., et al. 2003c, A\&A, 404, 1087

Kervella, P., Ségransan, D., \& Coudé du Foresto, V. 2004a, A\&A, 425, 1161

Kervella, P., Thévenin, F., Morel, P., et al. 2004b, A\&A, 413, 251 
Kjeldsen, H., \& Bedding, T. R. 2011, A\&A, 529, L8

Lopez, B., Petrov, R. G., \& Vannier, M. 2000, in SPIE Proc. 4006, ed. P. Léna, \& A. Quirrenbach, 407

Martić, M., Schmitt, J., Lebrun, J.-C., et al. 1999, A\&A, 351, 993

Martić, M., Lebrun, J.-C., Appourchaux, T., \& Korzennik, S. G. 2004, A\&A, 418, 295

Matter, A., Vannier, M., Morel, S., et al. 2010, A\&A, 515, A69

Matthews, J. M., Kuschnig, R., Guenther, D. B., et al. 2004, Nature, 430, 51

Mihalas, D., Dappen, W., \& Hummer, D. G. 1988, ApJ, 331, 815

Monnier, J. D. 2007, New Astron. Rev., 51, 604

Mosser, B., Bouchy, F., Martić, M., et al. 2008, A\&A, 478, 197

Mourard, D., Clausse, J. M., Marcotto, A., et al. 2009, A\&A, 508, 1073

Mozurkewich, D., Johnston, K. J., Simon, R. S., et al. 1991, AJ, 101, 2207

Nelson, G. D. 1980, ApJ, 238, 659

Nordlund, A. 1982, A\&A, 107, 1

Nordlund, A., \& Dravins, D. 1990, A\&A, 228, 155

Nordlund, Å., Stein, R. F., \& Asplund, M. 2009, Liv. Rev. Sol. Phys., 6, 2

Provencal, J. L., Shipman, H. L., Koester, D., Wesemael, F., \& Bergeron, P. 2002, ApJ, 568, 324

Provost, J., Berthomieu, G., Martić, M., \& Morel, P. 2006, A\&A, 460, 759
Quirrenbach, A. 2001, ARA\&A, 39, 353

Renard, S., Absil, O., Berger, J., et al. 2008, in SPIE Proc., 7013

Rodríguez-Pascual, P. M., González-Riestra, R., Schartel, N., \& Wamsteker, W. 1999, A\&AS, 139, 183

Schaeberle, J. M. 1896, AJ, 17, 37

Segransan, D., Beuzit, J.-L., Delfosse, X., et al. 2000, in SPIE Proc. 4006, ed. P. Léna, \& A. Quirrenbach, 269

Shao, M., Colavita, M. M., Hines, B. E., et al. 1988, A\&A, 193, 357

Short, C. I., \& Hauschildt, P. H. 2005, ApJ, 618, 926

Steffen, M. 1985, A\&AS, 59, 403

Strand, K. A. 1951, ApJ, 113, 1

ten Brummelaar, T. A., McAlister, H. A., Ridgway, S. T., et al. 2005, ApJ, 628, 453

van Belle, G. T. 2008, PASP, 120, 617

van Leeuwen, F. 2007, A\&A, 474, 653

Verner, G. A., Elsworth, Y., Chaplin, W. J., et al. 2011, MNRAS, 415, 3539

Wittkowski, M., Aufdenberg, J. P., \& Kervella, P. 2004, A\&A, 413, 711

Zhao, M., Monnier, J. D., ten Brummelaar, T., Pedretti, E., \& Thureau, N. D. 2008, in SPIE Conf. Ser. 7013

Zhao, M., Monnier, J. D., Che, X., et al. 2011, PASP, 123, 964 